\title{
Spitzer Space Telescope observations of bilobate comet 8P/Tuttle
}

\author{
O. Groussin ${ }^{1}$, P. L. Lamy ${ }^{2}$, M. S. P. Kelley ${ }^{3}$, I. Toth ${ }^{1,4}$, L. Jorda ${ }^{1}$, Y. R. Fernández ${ }^{5}$, and H. A. Weaver ${ }^{6}$ \\ ${ }^{1}$ Aix Marseille Univ, CNRS, CNES, LAM, Marseille, France \\ e-mail: olivier.groussin@lam.fr \\ ${ }^{2}$ Laboratoire Atmosphères, Milieux et Observations Spatiales, CNRS \& UVSQ, 11 bvd d'Alembert, 78280 Guyancourt, France \\ ${ }^{3}$ Department of Astronomy, University of Maryland, College Park, MD 20742-2421, USA \\ ${ }^{4}$ MTA CSFK Konkoly Observatory, H1121 Budapest, Konkoly Thege M. ut 15-17, Hungary \\ ${ }^{5}$ Department of Physics and Florida Space Institute, University of Central Florida, Orlando, FL 32816, USA \\ 6 The Johns Hopkins University Applied Physics Laboratory, Laurel, MD 20723, USA
}

Received 5 August 2019 / Accepted 7 November 2019

\begin{abstract}
Context. Comet 8P/Tuttle is a nearly isotropic comet whose physical properties are poorly known and might be different from those of ecliptic comets owing to their different origin. Two independent observations have shown that $8 \mathrm{P} /$ Tuttle has a bilobate nucleus.

Aims. Our goal is to determine the physical properties of the nucleus (size, shape, thermal inertia, and albedo) and coma (water and dust) of 8P/Tuttle.

Methods. We observed the inner coma of $8 \mathrm{P} /$ Tuttle with the infrared spectrograph and the infrared camera of the Spitzer Space Telescope. We obtained one spectrum $(5-40 \mu \mathrm{m})$ on 2 November 2007 and a set of 19 images at $24 \mu \mathrm{m}$ on 22-23 June 2008 sampling the rotational period of the nucleus. The data were interpreted using thermal models for the nucleus and the dust coma, and we considered two possible shape models of the nucleus derived from Hubble Space Telescope visible and Arecibo radar observations.

Results. We favor a model for the nucleus shape that is composed of two contact spheres with respective radii of $2.7 \pm 0.1 \mathrm{~km}$ and $1.1 \pm 0.1 \mathrm{~km}$ and a pole orientation with $\mathrm{RA}=285 \pm 12^{\circ}$ and $\mathrm{Dec}=+20 \pm 5^{\circ}$. The thermal inertia of the nucleus lies in the range $0-100 \mathrm{~J} \mathrm{~K}^{-1} \mathrm{~m}^{-2} \mathrm{~s}^{-1 / 2}$ and the $R$-band geometric albedo is $0.042 \pm 0.008$. The water production rate amounts to $1.1 \pm 0.2 \times$ $10^{28}$ molecules s ${ }^{-1}$ at $1.6 \mathrm{AU}$ from the Sun pre-perihelion, which corresponds to an active fraction of $\approx 9 \%$. At the same distance, the $\epsilon f \rho$ quantity amounts to $310 \pm 34 \mathrm{~cm}$, and it reaches $325 \pm 36 \mathrm{~cm}$ at $2.2 \mathrm{AU}$ post-perihelion. The dust grain temperature is estimated to be $258 \pm 10 \mathrm{~K}$, which is $37 \mathrm{~K}$ higher than the thermal equilibrium temperature at $1.6 \mathrm{AU}$. This indicates that the dust grains that contribute to the thermal infrared flux have a typical size of $\approx 10 \mu \mathrm{m}$. The dust spectrum exhibits broad emission around $10 \mu \mathrm{m}(1.5 \sigma$ confidence level) and $18 \mu \mathrm{m}$ ( $5 \sigma$ confidence level) that we attribute to amorphous pyroxene.
\end{abstract}

Key words. comets: general - comets: individual: 8P/Tuttle

\section{Introduction}

Comet 8P/Tuttle belongs to the family of nearly isotropic comets (NIC), following the classification of Levison \& Duncan (1997), and more precisely, to the family of Halley-type comets (HTC; Levison \& Duncan 1994). Compared with ecliptic comets (EC), little is known about the nucleus properties of NIC. While we have information on the properties of more than 200 EC (Lamy et al. 2004; Fernández et al. 2013), this is only the case for fewer than 30 NIC (Lamy et al. 2004). Owing to their different dynamical reservoirs, the Oort cloud for NIC (Levison et al. 2001) and the Kuiper belt for EC (Levison 1991), the question naturally arises as to whether these two populations have intrinsically different physical properties.

Prior to its last passage in January 2008 at only 0.25 AU from the Earth, the nucleus of comet 8P/Tuttle was thought to be very large. Licandro et al. (2000) derived a radius of $7.3 \mathrm{~km}$ from visible photometry at a heliocentric distance $\left(r_{\mathrm{h}}\right)$ of $6.3 \mathrm{AU}$, assuming a typical visible geometric albedo of 0.04 . This made $8 \mathrm{P} /$ Tuttle potentially one of the largest NIC after Hale-Bopp $(37 \mathrm{~km})$ and $109 \mathrm{P} /$ Swift-Tuttle $(13 \mathrm{~km})$ (Lamy et al. 2004). Visible observations performed in 2006 by Weissman et al. (2008), when the comet was at $r_{\mathrm{h}}=5.0 \mathrm{AU}$, also supported a large radius of $6.0 \mathrm{~km}$. However, radar observations performed by Harmon et al. (2010) in early January 2008 showed a very different picture: they revealed a bilobate shape. The two lobes were found to be elongated, with semi-axes of $2.1 \times 2.1 \times 2.9 \mathrm{~km}$ for the larger lobe and $1.6 \times 1.6 \times 2.1 \mathrm{~km}$ for the smaller lobe. This implies a much smaller nucleus than was originally found. Observations in the visible performed by Lamy et al. (2008a) with the Hubble Space Telescope (HST) on 10-11 December 2007 during 12 HST visits extending over a 28 -h time interval also indicated a small nucleus with a radius of $3.0 \mathrm{~km}$. Harmon et al. (2010) and Lamy et al. (2008a) derived a rotation period of $11.4 \mathrm{~h}$. From millimeter observations with the Plateau de Bure interferometer, Boissier et al. (2011) obtained an upper limit for the nucleus thermal inertia of $10 \mathrm{~J} \mathrm{~K}^{-1} \mathrm{~m}^{-2} \mathrm{~s}^{-1 / 2}$.

The water production rate of comet $8 \mathrm{P} /$ Tuttle was measured close to perihelion ( $r_{\mathrm{h}}=1.03$ AU on 27 January 2008) by several observers. Biver et al. (2008) derived a water production rate of $4.0 \times 10^{28}$ molecules $\mathrm{s}^{-1}$ from millimeter observations (IRAM) between 29 December 2007 and 2 January 2008, when 8P/Tuttle was at $r_{\mathrm{h}}=1.10-1.12$ AU. Barber et al. (2009) observed 8P/Tuttle in the near-infrared with the United Kingdom Infrared Telescope (UKIRT) on 3 January $2008\left(r_{\mathrm{h}}=1.09 \mathrm{AU}\right)$ and derived a water production rate of 
$1.4 \pm 0.1 \times 10^{28}$ molecules $\mathrm{s}^{-1}$. Lovell \& Howell (2008) derived a water production rate of $1.8 \times 10^{28}$ molecules $\mathrm{s}^{-1}$ from radio observations (Arecibo and Green Bank) on 15 January 2008, at $r_{\mathrm{h}}=1.04 \mathrm{AU}$. Lippi et al. (2008) derived a water production rate of 5.4-6.0 $\times 10^{28}$ molecules $\mathrm{s}^{-1}$ from near-infrared observations using the CRyogenic high-resolution InfraRed Echelle Spectrograph (CRIRES) at the ESO Very Large Telescope (VLT) on 27 January 2008, at $r_{\mathrm{h}}=1.03$ AU. With the same instrument, but a few days later (28 January 2008-4 February 2008), Bockelée-Morvan et al. (2008) derived a water production rate of 3.9-4.4 × $10^{28}$ molecules s ${ }^{-1}$, at $r_{\mathrm{h}}=1.03 \mathrm{AU}$.

To summarize, the water production rate of comet $8 \mathrm{P} /$ Tuttle close to perihelion lies in the range $1.4-6.0 \times 10^{28}$ molecules $\mathrm{s}^{-1}$. For a radius of $3.0 \mathrm{~km}$, this corresponds to a surface active area in the range $3-15 \%$, derived from the water production rate of a spherical nucleus made of water ice only, located at perihelion, and assuming a temperature distribution similar to that of the standard thermal model with a beaming factor of 1 (Lebofsky et al. 1986). For other gas species that are not relevant to this paper, we refer to A'Hearn et al. (1995), Böhnhardt et al. (2008), Bonev et al. (2008), Jehin et al. (2009), and Kobayashi et al. (2010).

Two determinations of the $A f \rho$ quantity of comet $8 \mathrm{P} /$ Tuttle are available: $110 \mathrm{~cm}$ by A'Hearn et al. (1995) when the comet was close to perihelion in August 1994, and $32 \mathrm{~cm}$ by Schleicher (2007) during the interval 3-5 December 2007 at $r_{\mathrm{h}}=1.3 \mathrm{AU}$. These values are lower than for other comets (A'Hearn et al. 1995), which likely indicates a paucity of submicron-size dust particles.

The peculiar bilobate nature of $8 \mathrm{P} /$ Tuttle was relatively unique when it was discovered in 2008. The only other questionable examples at that time were 1P/Halley based on its "central depression" (Keller et al. 1987) and 19P/Borrelly (Soderblom et al. 2002). Since then, two other cometary nuclei have been confirmed to be bilobate: 103P/Hartley 2 (A'Hearn et al. 2011) and 67P/Churyumov-Gerasimenko (Sierks et al. 2015). Overall, four of the six comets for which we have spatially resolved images of their nucleus have a bilobate shape, which therefore seems a common shape among cometary nuclei.

The aim of this paper is to present the results of our Spitzer Space Telescope (SST) observations of comet 8P/Tuttle, which were performed on 2 November 2007 with the infrared spectrograph (IRS) instrument and during 22-23 June 2008 with the infrared camera (MIPS) instrument, in order to determine the physical properties of its nucleus (size, shape, thermal inertia, and albedo) and the activity level of its coma (water and dust). In particular, using thermal infrared observations, we estimate the size of the nucleus, independently of its geometric albedo.

\section{Observations with the Spitzer Space Telescope}

\subsection{IRS and MIPS observations}

The orbital elements of 8P/Tuttle are given in Table 1. Only two visibility windows of about three months each were available to observe comet $8 \mathrm{P} /$ Tuttle with the SST during cycle 4 (June 2007-June 2008) because of the restriction on solar elongation $\left(80-120^{\circ}\right)$. The first window, from 4 October 2007 to 24 January 2008, covered the pre-perihelion phase from $r_{\mathrm{h}}=1.9$ to $1.03 \mathrm{AU}$, with an increasing phase angle from 32 to $75^{\circ}$. The second window, from 4 April 2008 to 30 June 2008, covered the post-perihelion phase from $r_{\mathrm{h}}=1.5$ to $2.3 \mathrm{AU}$, with a decreasing phase angle from 40 to $22^{\circ}$. At the time of proposal preparation, the best size estimate of the nucleus radius was $7.3 \mathrm{~km}$
Table 1. Orbital elements of comet 8P/Tuttle from the JPL Horizons website $^{1}$ for the 27 January 2008 perihelion passage.

\begin{tabular}{cccccc}
\hline \hline $\begin{array}{c}q \\
{[\mathrm{AU}]}\end{array}$ & $\begin{array}{c}Q \\
{[\mathrm{AU}]}\end{array}$ & $e$ & $\begin{array}{c}i \\
{\left[{ }^{\circ}\right]}\end{array}$ & $\begin{array}{c}P \\
{[\mathrm{yr}]}\end{array}$ & $T_{\mathrm{J}}$ \\
\hline 1.03 & 10.4 & 0.82 & 55 & 13.6 & 1.6 \\
\hline
\end{tabular}

Notes. The table lists: perihelion distance $(q)$, aphelion distance $(Q)$, eccentricity $(e)$, inclination $(i)$, orbital period $(P)$, and Tisserand parameter with respect to Jupiter $\left(T_{\mathrm{J}}\right)$.

(Licandro et al. 2000), so that we anticipated a very high flux. This flux was expected to saturate the MIPS $24 \mu \mathrm{m}$ detector during almost the entire first window and to saturate the IRS detector close to perihelion. As a consequence, the scheduled window was carefully selected to maximize the signal-to-noise ratio $(\mathrm{S} / \mathrm{N})$ on the nucleus without saturating the MIPS and IRS detectors. Because of further additional constraints on the phase angle, we ultimately decided on the following observing strategy: (i) perform the IRS observations on 2 November 2007, before the expected flux reached the saturation limit, and (ii) perform the MIPS observations on 22-23 June 2008, at low phase angle after the expected flux dropped below the saturation limit. Table 2 summarizes the IRS and MIPS observations.

At the time of the IRS observations, 8P/Tuttle was at $r_{\mathrm{h}}=1.61 \mathrm{AU}$, a distance from the SST of $1.32 \mathrm{AU}$, and a solar phase angle of $39^{\circ}$. We used IRS in the low-resolution mode $(R=\lambda / \Delta \lambda \approx 64-128)$, which covers the wavelength range 5.2$38.0 \mu \mathrm{m}$ in four long-slit segments: the short wavelength second order (SL2, from 5.2 to $8.5 \mu \mathrm{m}$ ), the short wavelength first order (SL1, from 7.4 to $14.2 \mu \mathrm{m}$ ), the long wavelength second order (LL2, from 14.0 to $21.5 \mu \mathrm{m}$ ), and the long wavelength first order (LL1, from 19.5 to $38.0 \mu \mathrm{m}$ ). We acquired three spectra with an integration time of $18.9 \mathrm{~s}$ for each segment, that is, a total integration time of $56.7 \mathrm{~s}$ per segment. The pointing of the target was performed using the ephemeris derived from the JPL Horizons website ${ }^{1}$. We were unable to use the peak-up cameras at the time of observation because of saturation issues. However, because the SST pointing error is only $\approx 1^{\prime \prime}$ (smaller than the slit width) and because the ephemeris was even more accurate, the peak-up cameras were unnecessary. The same sequence was repeated two days later, on 4 November 2007, at the same RA and Dec as the original observations, to obtain shadow observations in order to properly subtract the sky background.

At the time of the MIPS observations, 8P/Tuttle was at $r_{\mathrm{h}}=2.24 \mathrm{AU}$, a distance from the SST of $1.58 \mathrm{AU}$, and a solar phase angle of $23^{\circ}$. We used the MIPS imaging capabilities at 24 and $70 \mu \mathrm{m}$ to take observations centered on the nucleus. At $24 \mu \mathrm{m}$, we performed 20 observations, with a common integration time of $48.2 \mathrm{~s}$. Each observation consists of 14 dithered frames mosaicked together (Sect. 2.3). The MIPS $24 \mu$ m detector works at an effective wavelength of $23.7 \mu \mathrm{m}$ with a pixel scale of $2.55^{\prime \prime} /$ pixel. At $70 \mu \mathrm{m}$, we performed 4 observations centered on the nucleus, with a common integration time of $37.7 \mathrm{~s}$. Each observation consists of 12 dithered frames mosaicked together (Sect. 2.3). The MIPS $70 \mu \mathrm{m}$ detector works at an effective wavelength of $71.0 \mu \mathrm{m}$ with a pixel scale of $9.96^{\prime \prime}$ pixel $^{-1}$. At the time of proposal preparation, the rotation period of the nucleus was unknown. To minimize the amount of requested observing time and to still maintain a reasonable chance of obtaining the light-curve extrema, the 20 observations at $24 \mu \mathrm{m}$ were

https://ssd.jpl.nasa.gov/horizons.cgi 
Table 2. IRS and MIPS observations.

\begin{tabular}{|c|c|c|c|c|c|c|c|}
\hline Instrument & $\begin{array}{c}\lambda \\
{[\mu \mathrm{m}]}\end{array}$ & $\begin{array}{l}\text { Date } \\
\text { [UT] }\end{array}$ & $\begin{array}{c}r_{\mathrm{h}} \\
{[\mathrm{AU}]}\end{array}$ & $\begin{array}{c}\Delta \\
{[\mathrm{AU}]}\end{array}$ & $\begin{array}{c}\alpha \\
{\left[{ }^{\circ}\right]}\end{array}$ & $\begin{array}{l}\text { Nucleus flux } \\
{[\mathrm{mJy}]}\end{array}$ & $\begin{array}{c}\epsilon f \rho \\
{[\mathrm{cm}]}\end{array}$ \\
\hline IRS & $5.2-38.0$ & 20071102.76 & 1.606 & 1.322 & 39.4 & N/A & $310 \pm 34$ \\
\hline MIPS & 23.7 & 20080622.49 & 2.243 & 1.579 & 23.5 & - & $326 \pm 36$ \\
\hline MIPS & 23.7 & 20080622.52 & 2.243 & 1.579 & 23.5 & $110 \pm 6$ & $329 \pm 36$ \\
\hline MIPS & 23.7 & 20080622.53 & 2.243 & 1.579 & 23.5 & $100 \pm 5$ & $327 \pm 36$ \\
\hline MIPS & 23.7 & 20080622.58 & 2.244 & 1.580 & 23.5 & $85 \pm 4$ & $327 \pm 36$ \\
\hline MIPS & 23.7 & 20080622.62 & 2.244 & 1.580 & 23.5 & $105 \pm 5$ & $326 \pm 36$ \\
\hline MIPS & 23.7 & 20080622.64 & 2.244 & 1.580 & 23.5 & $108 \pm 5$ & $328 \pm 36$ \\
\hline MIPS & 23.7 & 20080622.66 & 2.244 & 1.580 & 23.5 & $110 \pm 6$ & $326 \pm 36$ \\
\hline MIPS & 23.7 & 20080622.70 & 2.245 & 1.580 & 23.4 & $122 \pm 6$ & $328 \pm 36$ \\
\hline MIPS & 23.7 & 20080622.74 & 2.245 & 1.581 & 23.4 & $130 \pm 7$ & $328 \pm 36$ \\
\hline MIPS & 23.7 & 20080622.79 & 2.246 & 1.581 & 23.4 & $120 \pm 6$ & $327 \pm 36$ \\
\hline MIPS & 23.7 & 20080622.83 & 2.246 & 1.581 & 23.4 & $110 \pm 6$ & $324 \pm 36$ \\
\hline MIPS & 23.7 & 20080622.85 & 2.246 & 1.582 & 23.4 & $105 \pm 5$ & $326 \pm 36$ \\
\hline MIPS & 23.7 & 20080622.87 & 2.247 & 1.582 & 23.4 & $115 \pm 6$ & $326 \pm 36$ \\
\hline MIPS & 23.7 & 20080622.92 & 2.247 & 1.582 & 23.4 & $125 \pm 6$ & $325 \pm 36$ \\
\hline MIPS & 23.7 & 20080622.95 & 2.247 & 1.582 & 23.4 & $113 \pm 6$ & $327 \pm 36$ \\
\hline MIPS & 23.7 & 20080622.97 & 2.248 & 1.582 & 23.4 & $110 \pm 6$ & $325 \pm 36$ \\
\hline MIPS & 23.7 & 20080622.99 & 2.248 & 1.582 & 23.4 & $108 \pm 5$ & $324 \pm 36$ \\
\hline MIPS & 23.7 & 20080623.03 & 2.248 & 1.583 & 23.4 & $104 \pm 5$ & $322 \pm 36$ \\
\hline MIPS & 23.7 & 20080623.08 & 2.249 & 1.583 & 23.4 & $100 \pm 5$ & $322 \pm 36$ \\
\hline MIPS & 23.7 & 20080623.12 & 2.249 & 1.583 & 23.4 & $109 \pm 5$ & $322 \pm 36$ \\
\hline MIPS & 71.0 & 20080622.49 & 2.243 & 1.579 & 23.5 & - & - \\
\hline MIPS & 71.0 & 20080622.62 & 2.244 & 1.580 & 23.5 & - & - \\
\hline MIPS & 71.0 & 20080622.83 & 2.246 & 1.581 & 23.4 & - & - \\
\hline MIPS & 71.0 & 20080622.95 & 2.247 & 1.582 & 23.4 & - & - \\
\hline
\end{tabular}

Notes. For each observation, we list the instrument, the observation wavelength $(\lambda)$, the starting date of the observation, the heliocentric distance $\left(r_{\mathrm{h}}\right)$, the distance to the SST $(\Delta)$, the phase angle $(\alpha)$, the infrared flux of the nucleus, and the dust $\epsilon f \rho$ quantity (see text for details).

distributed unevenly over $15 \mathrm{~h}$ and separated by either 0.5 or $1.0 \mathrm{~h}$. The 4 observations at $70 \mu \mathrm{m}$ were likewise distributed unevenly over $15 \mathrm{~h}$ and inserted between the $24 \mu \mathrm{m}$ observations. The same sequence was repeated one day later, on 24 June 2008, at the same RA and Dec as the original observations, to secure shadow observations.

Details about the SST can be found in Werner et al. (2004) and in the Spitzer observer's manual ${ }^{2}$. More information on the instruments can be found in Houck et al. (2004) for IRS and in Rieke et al. (2004) for MIPS.

\subsection{IRS data reduction}

Spectra of comet $8 \mathrm{P} /$ Tuttle acquired with the IRS instrument were initially processed and calibrated with the Spitzer Science Center IRS pipeline (version S17.0.4). We subtracted a shadow observation from each target observation to remove the sky background. Some residual background flux still remained in the 2D spectral images. Therefore a second subtraction was performed using median-combined sky frames taken contemporaneously with the comet. This removes any zodiacal light or instrument background that is not fully accounted for in the shadow observations. Bad pixels were identified and replaced through nearest-neighbor interpolation, or were ignored altogether.

We extracted spectra from the 2D images using the Spitzer Science Center SPICE software ${ }^{3}$. Our synthetic apertures,

2 http://ssc.spitzer.caltech.edu/documents/som/

3 SPICE is available at http://ssc.spitzer.caltech.edu/ centered on the peak of the source, used the default point-source aperture widths, which vary with $\lambda / \lambda_{0}: 4.0$ pixels at $\lambda_{0}=6.0 \mu \mathrm{m}$ (SL2) and 8.0 pixels at $\lambda_{0}=12.0 \mu \mathrm{m}$ (SL1) for the short-low extractions, and 4.3 pixels at $\lambda_{0}=16.0 \mu \mathrm{m}$ (LL2) and 7.2 pixels at $\lambda_{0}=27.0 \mu \mathrm{m}$ (LL1) for long-low. Finally, to improve the $\mathrm{S} / \mathrm{N}$, we computed the median spectrum of our three spectra.

The goal of our observations is to measure the spectrum of the nucleus, therefore we did not apply any extended source calibrations to the extracted data in order to preserve the spectral shape of the emission from the unresolved nucleus. As we show in Sect. 5.1, the nucleus contributes $\approx 50 \%$ of the total signal in SL mode and $\approx 25 \%$ of the total signal in LL mode.

\subsection{MIPS data reduction}

The images acquired with MIPS were processed with the Spitzer Science Center pipeline (version S18.0.2), producing individual basic calibrated data (BCD) frames. We subtracted a shadow observation from each target observation to remove the sky background. The BCD images were subsequently mosaicked in the rest frame of the comet with the MOPEX software (version 16.3.7). Bad pixels, that is, pixels that are permanently damaged or affected by cosmic rays, were ignored during the mosaicking step. Figure 1 shows an example of a calibrated image at $24 \mu \mathrm{m}$ and $70 \mu \mathrm{m}$.

The coma, dust tail, and first Airy ring are visible in the $24 \mu \mathrm{m}$ image, indicating a high nucleus-to-coma ratio in the central pixel. We extracted the nucleus signal using our standard 

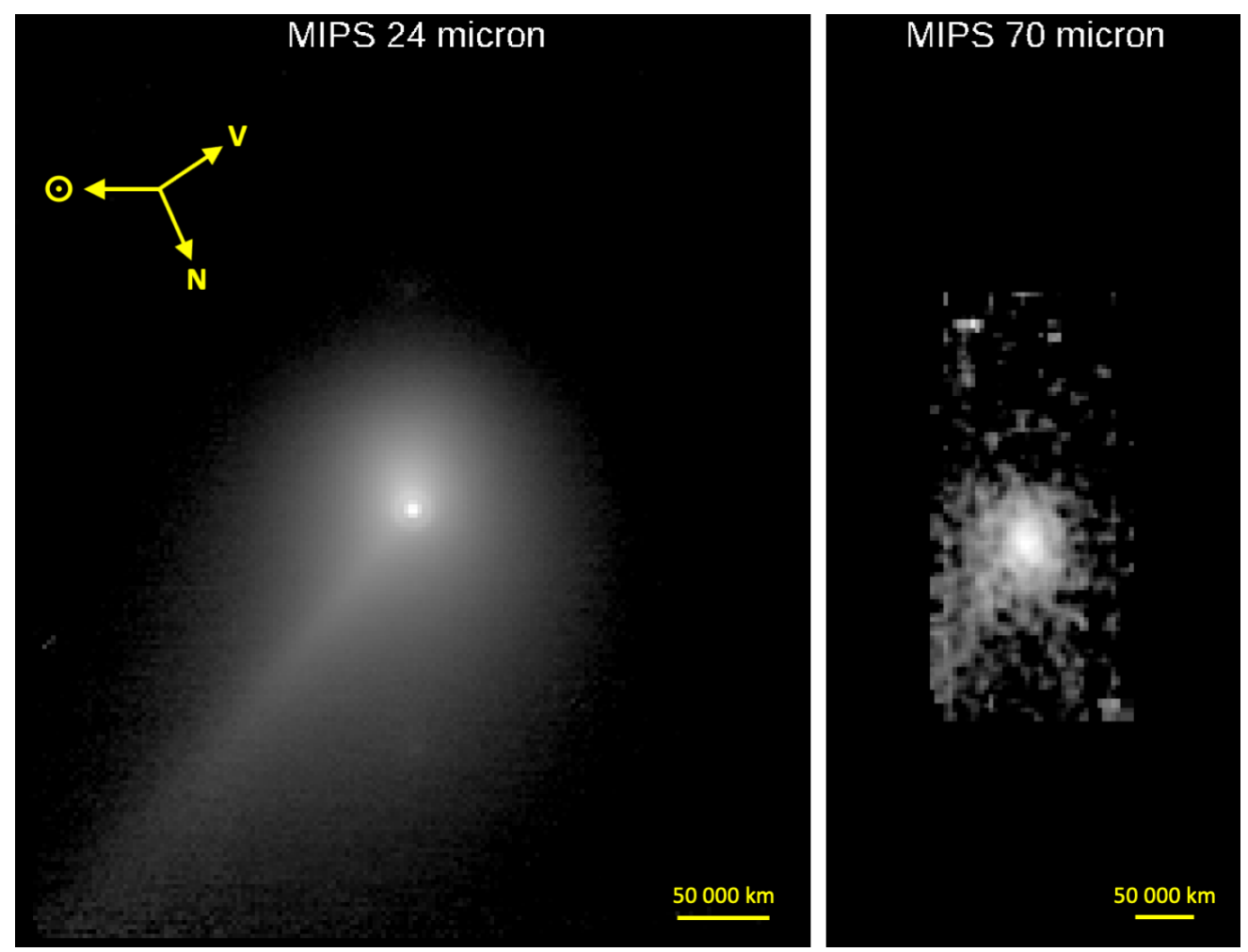

Fig. 1. MIPS infrared calibrated images of comet 8P/Tuttle taken on 22 June 2008 ( $24 \mu \mathrm{m}$ on the left, $70 \mu \mathrm{m}$ on the right). The images are displayed with a logarithmic stretch. The $24 \mu \mathrm{m}$ image has a size of $176 \times 194$ pixels with a projected pixel size of about $2450 \mathrm{~km}$. The yellow arrows indicate the direction to the Sun $(\odot)$, celestial North $(\mathrm{N})$, and the velocity vector $(\mathrm{V})$. The $70 \mu \mathrm{m}$ image has a size of $46 \times 95$ pixels with a projected pixel size of about $3840 \mathrm{~km}$. The coma, dust tail, and first Airy ring are visible in the $24 \mu \mathrm{m}$ image. The $70 \mu \mathrm{m}$ image is extremely noisy and unsuitable for nucleus extraction (see text for detail).

method of fitting a parametric model of the expected surface brightness to the observed images, as implemented for instance in MIPS observations of comet 67P/Churyumov-Gerasimenko by Lamy et al. (2008b). A 2D brightness array was constructed, which superimposes an unresolved nucleus represented by a Dirac function and a simple coma model that follows the canonical $1 / \rho$ radial variation $(\rho$ is the projected distance from the nucleus), both convolved by the point spread function (PSF) of the telescope. The PSFs were generated with the STINY$\mathrm{TIM}^{4}$ tool, following the procedure described in Lamy et al. (2008b). The model images were generated on a finer grid than the original MIPS pixel, with a resampling factor of 10 . The fit to the real images was performed after integrating the model over $10 \times 10$ sub-pixels to recover the original pixel of the MIPS. The fits to the observations were performed on azimuthally averaged radial profiles excluding the sector affected by the tail and led to the determination of the respective scaling factors of the nucleus and coma models. As shown in one example given in Fig. 2, the fits were satisfactory up to a distance $\rho=4$ pixels $(9800 \mathrm{~km}$ at $1.58 \mathrm{AU}$ ) with residuals of typically $1 \%$. The total signal in the central pixel is dominated by the coma, and the nucleus contribution amounts to a fraction of only $\approx 25 \%$ of the total signal. This is sufficient for a robust nucleus extraction, however, as justified by Hui \& Li (2018). The derived fluxes for the nucleus are given in Table 2 for each image. The typical $1 \sigma$ error is $5 \%$. We were unable to extract the nucleus in the first image because the fit

\footnotetext{
4 STINYTIM is available at https://irsa.ipac.caltech.edu/ data/SPITZER/docs/dataanalysistools/tools/contributed/ general/stinytim/
}

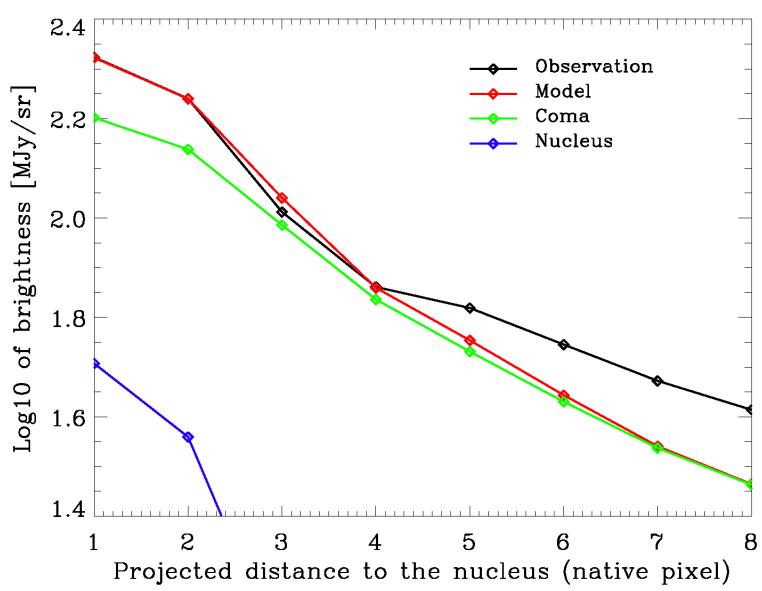

Fig. 2. Azimuthal average profile of the central coma of comet 8P/Tuttle, observed by the MIPS instrument at $24 \mu \mathrm{m}$, and compared with a photometric model comprising a separate contribution from the nucleus and the coma. In the central 2 pixels, used to derive the nucleus contribution, the fit is excellent and the model and the observation are identical.

of the model to the average radial profile was very poor and the resulting nucleus flux was abnormally low ( $<50 \mathrm{mJy})$ compared with the values for the other 19 MIPS images.

The $\mathrm{S} / \mathrm{N}$ of $\approx 6$ in the $70 \mu \mathrm{m}$ images was too low to extract the nucleus. Its contribution in the central pixel was estimated to be $\approx 10 \%$ of the total flux on the basis of the $24 \mu \mathrm{m}$ image, using geometrical considerations. This results from the larger field of view at $70 \mu \mathrm{m}$, which translates into a larger contribution from 
the coma, whereas the contribution of the nucleus remains the same. In comparison, the $24 \mu \mathrm{m}$ images have an $\mathrm{S} / \mathrm{N}$ of $\approx 200$.

\section{Thermal models for nucleus and coma}

The IRS spectrum is a combination of the thermal flux from the nucleus and the dust and gas coma. Thermal models of the nucleus and the coma are therefore required to estimate their respective contribution to the total spectral energy distribution (SED). For MIPS, only a thermal model for the nucleus is required because its flux can be extracted from the overall signal (nucleus + coma), as explained in Sect. 2.3.

\subsection{Thermal model for the nucleus}

\subsubsection{Shape model for the nucleus}

The thermal model for the nucleus first requires a shape model. For 8P/Tuttle, we cannot make the usual assumption of a spherical nucleus because there is evidence for a bilobate shape. Currently, two different shape models for the nucleus of comet 8P/Tuttle are available. The first (hereafter HST shape model) is derived from the inversion of a visible light curve obtained by the HST (Lamy et al. 2008a), and the second (hereafter radar shape model) is derived from radar observations (Harmon et al. 2010). The two shape models correspond to a contact binary, but they differ in the shape and size of the primary and secondary (Table 3 and Fig. 3).

The HST shape model consists of two spheres in contact with a ratio of 2.3 between their radii. The absolute scale of this model is not constrained because it depends on the albedo. For a typical geometric albedo of 0.04 ( $R$ band), the radius of the two spheres amounts to $2.8 \mathrm{~km}$ and $1.2 \mathrm{~km}$, respectively. The pole orientation for the HST shape model defined by RA $=285 \pm 12^{\circ}$ and Dec $=+20 \pm 5^{\circ}$ yields an aspect angle (defined as the angle between the spin vector and the comet-SST vector) of $92^{\circ}$ on 2 November 2007 (IRS) and $65^{\circ}$ on 22-23 June 2008 (MIPS).

The radar shape model consists of two prolate ellipsoids in contact, aligned along their long axis. The semi-axes are $a=2.06 \mathrm{~km}, b=2.06 \mathrm{~km}$ and $c=2.88 \mathrm{~km}$ for the primary, and $a=1.64 \mathrm{~km}, b=1.64 \mathrm{~km}$ and $c=2.13 \mathrm{~km}$ for the secondary. The uncertainty is $10 \%$ on these values. The pole axis is perpendicular to the long axis. The radar observations constrain the pole orientation to lie within a cone corresponding to a projection angle of $55 \pm 7^{\circ}$ from the observer. However, we calculated that inside this cone, the solution that best fits the HST light curve corresponds to $\mathrm{RA}=268 \pm 5^{\circ}$ and $\mathrm{Dec}=-16 \pm 2^{\circ}$, which gives an aspect angle of $60^{\circ}$ on 2 November 2007 (IRS) and $104^{\circ}$ on 22-23 June 2008 (MIPS).

The separation is $40^{\circ}$ between the pole directions given by the two models. However, both have a rotational period of $11.4 \mathrm{~h}$.

\subsubsection{Thermal model}

We implemented our thermal model for the nucleus, which has previously been extensively described in several past articles (e.g., Groussin et al. 2004; Lamy et al. 2008c), so that we limit ourselves to a short description here.

We consider the two shape models with their respective pole orientation. For each model, the surface of the nucleus was divided into 2560 facets, and for each facet, we solved for the surface energy balance between the flux received from the Sun, the reradiated flux, and the heat conduction into the nucleus. As we show below, the active fraction of $8 \mathrm{P} /$ Tuttle is restricted to $\approx 9 \%$ of its surface (Sect. 5.5). Like for comet 9P/Tempel 1, which has an active fraction of $9 \%$ (Lisse et al. 2005), the sublimation of water ice can be neglected in the energy balance for the calculation of the thermal flux that is emitted from the nucleus surface (Groussin et al. 2007). As the nucleus rotates around its spin axis, the illumination changes and the heat conduction equation is computed for each facet considering a 1D time-dependent equation. The projected shadows were taken into account. We used a time step of $\approx 12 \mathrm{~s}$, which is small enough compared with the rotation period $(\approx 11.4 \mathrm{~h})$ to ensure relaxation of the numerical solution in a few dozen rotations (depending on the thermal inertia). As a result, we obtained the temperature $T_{i}$ of each facet as a function of time over one period of rotation.

From this surface temperature distribution, we calculated the infrared flux received by the observer from each facet as a function of time and wavelength, 5-40 $\mu \mathrm{m}$ for IRS and $24 \mu \mathrm{m}$ for MIPS. The total flux $F_{\text {nucl }}$ is then the sum of all individual fluxes of each facet of the shape model (Eq. (1)). When the thermal inertia is not null and because the phase angle is not negligible for the IRS $\left(39^{\circ}\right)$ and MIPS $\left(24^{\circ}\right)$ observations, the infrared flux depends on the solution adopted for the rotation, that is, prograde $(\mathrm{P})$ or retrograde $(\mathrm{R})$. Owing to the lack of information on this point, we studied both cases,

$F_{\text {nucl }}(\lambda)=\gamma \sum_{i=1}^{n} \epsilon B\left(\lambda, T_{i}\right) \mathrm{d} \Omega_{i}$.

\subsubsection{Parameters of the thermal model for the nucleus}

Our model has six free parameters: the infrared emissivity $\epsilon$, the phase integral $q$, the scaling coefficient for the nucleus flux $\gamma$ (this corresponds to a scaling coefficient $\sqrt{\gamma}$ for the shape model), the geometric albedo $p_{\mathrm{v}}$, the beaming factor $\eta$, and the thermal inertia $I$. We considered that three of these six parameters, $\epsilon, q$, and $p_{\mathrm{v}}$, can be reasonably assumed, but the other three, $\gamma, \eta$, and $I$, must be constrained by the observations.

We adopted a value of 0.95 for the thermal emissivity $\epsilon$, which is the mid-point of the values that are typically quoted in the literature (0.9-1.0).

The phase integral $q$ measures the angular dependence of the scattered radiation. We chose $q=0.27$, the value found for 19P/Borrelly by Buratti et al. (2004). We adopted a geometric albedo $p_{\mathrm{v}}=0.04$, a typical value for cometary nuclei (Lamy et al. 2004). The choice of $q$ and $p_{\mathrm{v}}$ has a negligible influence on the size determination as long as the product $p_{\mathrm{v}} q$ remains in the range $0.0-0.1$, which is the case for all cometary nuclei.

The beaming factor $\eta$ follows the strict definition given by Lagerros (1998) and therefore only reflects the influence of surface roughness, which produces an anisotropic thermal emission. Theoretically, $\eta$ ranges from 0 to 1 , but in practice, it is larger than 0.7 to avoid unrealistic roughness (Lagerros 1998). It differs from the factor $\eta$ used in the standard thermal model (STM; Lebofsky \& Spencer 1989) or in the near-Earth asteroid thermal model (NEATM; Harris 1998), where $\eta$ is a combination of roughness and thermal inertia and thus can be larger than one. In this study, we considered four values for $\eta$ : 0.7, 0.8, 0.9, and 1.0 .

We considered several values for thermal inertia: $I=0,50$, $100,200,400$, and $800 \mathrm{~J} \mathrm{~K}^{-1} \mathrm{~m}^{-2} \mathrm{~s}^{-1 / 2}$. This covers more than the range $0-350 \mathrm{~J} \mathrm{~K}^{-1} \mathrm{~m}^{-2} \mathrm{~s}^{-1 / 2}$ that has been found for comets (see the review paper of Groussin et al. 2019).

The parameter $\gamma$ scales the nucleus flux to match the data, and directly scales the size of the nucleus set by the shape model. As a consequence, it can be independently determined for each combination of $\eta$ and $I$. 
Table 3. HST and radar shape models.

\begin{tabular}{|c|c|}
\hline \multicolumn{2}{|c|}{ HST shape model (Lamy et al. 2008a) } \\
\hline Shape & Two spheres in contact \\
\hline Largest sphere (radius) & $2.8 \mathrm{~km}$ \\
\hline Smallest sphere (radius) & $1.2 \mathrm{~km}$ \\
\hline Pole orientation & $\mathrm{RA}=285 \pm 12^{\circ}$ and $\mathrm{Dec}=+20 \pm 5^{\circ}$ \\
\hline \multicolumn{2}{|c|}{ Radar shape model (Harmon et al. 2010) } \\
\hline Shape & Two prolate spheroids in contact, aligned along their long axis \\
\hline Largest spheroid (semi-axis) & $2.06 \times 2.06 \times 2.88 \mathrm{~km}$ \\
\hline Smallest spheroid (semi-axis) & $1.64 \times 1.64 \times 2.13 \mathrm{~km}$ \\
\hline Pole axis & Perpendicular to the long axis \\
\hline Pole orientation & $\mathrm{RA}=268 \pm 5^{\circ}$ and $\mathrm{Dec}=-16 \pm 2^{\circ}$ (this work) \\
\hline
\end{tabular}
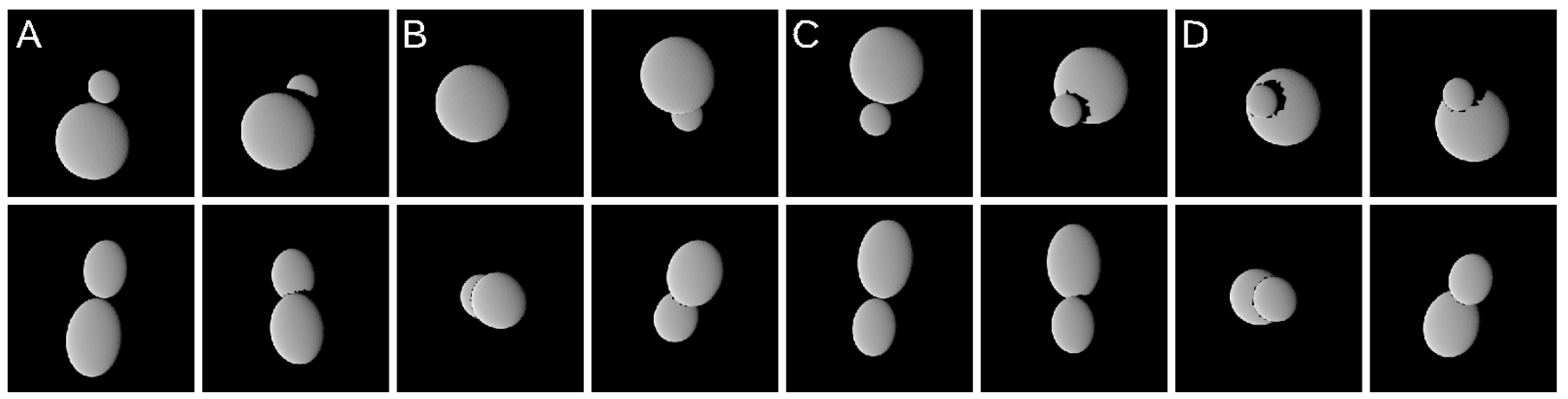

Fig. 3. HST shape model (top row) and radar shape model (bottom row) as viewed by SST on 22-23 June 2008. Letters A, B, C, and D correspond to different times (extrema) during the rotation and are connected to Fig. 5.

\subsection{Thermal model for the dust coma}

In addition to the thermal model for the nucleus, the interpretation of the IRS spectra requires a thermal model for the dust coma in order to estimate the SED. There are a multitude of possibilities for the dust SED based on many choices of grain size distribution, grain composition, and grain structure. Moreover, our IRS spectrum (as we show) has a limited amount of compositional diagnostics in it that could help us to independently constrain the dust grain properties. To make the problem tractable, we therefore adopted a simple graybody model, as given by Eq. (2),

$F_{\text {coma }}(\lambda)=\mathcal{A} \frac{\epsilon_{\text {dust }}}{\Delta^{2}} B\left(\lambda, T_{\text {dust }}\right)\left(\frac{\lambda}{\lambda_{0}}\right)^{p}$,

where $F_{\text {coma }}(\lambda)$ is the thermal flux (Jy) at the wavelength $\lambda(\mu \mathrm{m})$, $\mathcal{A}$ is the dust cross-section in the field of view $\left(\mathrm{m}^{2}\right), \epsilon_{\text {dust }}$ is the dust emissivity (we assume 0.95 ), $\Delta$ is the observer-comet distance $(\mathrm{m})$, and $B\left(\lambda, T_{\text {dust }}\right)$ is the Planck function at temperature $T_{\text {dust }}(\mathrm{K})$. The two unknowns are $\mathcal{A}$ and $T_{\text {dust }}$.

The factor $\left(\lambda / \lambda_{0}\right)^{p}$ is required for the aperture correction of the coma (that is an extended source), which was not taken into account in the data reduction (Sect. 2.2). This correction is purely geometrical. We used $\lambda_{0}=5.0 \mu \mathrm{m}$ for the SL mode and $\lambda_{0}=14.0 \mu \mathrm{m}$ for the LL mode. The power $p$ was used to convert the rectangular aperture of the IRS slit into an equivalent circular aperture, assuming a canonical $1 / \rho$ radial brightness profile for the coma. For the SL and LL modes, we computed $p=0.37$. This means, for example, that without the aperture correction, the coma flux is $46 \%$ higher at $14.0 \mu \mathrm{m}$ than at $5.0 \mu \mathrm{m}$ in the SL mode, or $40 \%$ higher at $35.0 \mu \mathrm{m}$ than at $14.0 \mu \mathrm{m}$ in the LL mode.

\section{MIPS data analysis}

\subsection{Adjusting the model to the data}

The thermal infrared light curve of the nucleus of comet $8 \mathrm{P} /$ Tuttle derived from the MIPS observations (Table 2) is plotted in Fig. 4. It was phase-folded using a rotation period of $11.4 \mathrm{~h}$. The light curve is double peaked, and one minimum is deeper than the other, in agreement with a contact binary and similar to the HST visible light curve (Lamy et al. 2008a).

As explained above, we calculated a synthetic thermal light curve for different values of $\eta$ and $I$ for each shape model (HST and radar). For each combination of $\eta$ and $I$, we adjusted the synthetic light curve to the data by tuning the scaling factor $\gamma$ and the phase. The best fit was determined by minimizing the chi-square value. Results are given in Table 4 and are illustrated in Fig. 5. The uncertainty on $\gamma$ is $\approx 5 \%$.

\subsection{HST and radar shape models}

The HST shape model provides a better fit to the data than the radar shape model, whatever the combination of $\eta$ and $I$, and regardless of whether the rotation is prograde or retrograde. The minimum chi-square value for the radar shape model $\left(\chi^{2}=108.5\right.$ for $\eta=0.7, I=400 \mathrm{~J} \mathrm{~K}^{-1} \mathrm{~m}^{-2} \mathrm{~s}^{-1 / 2}$, retrograde rotation) is $\approx 3$ times higher than the maximum chi-square value for the HST shape model $\left(\chi^{2}=37.2\right.$ for $\eta=1.0, I=0 \mathrm{~J} \mathrm{~K}^{-1} \mathrm{~m}^{-2} \mathrm{~s}^{-1 / 2}$, prograde rotation), and in most cases, the chi-square values differ by a factor $>5$ between the two shape models.

For the radar shape model, the $10 \%$ uncertainty on the nucleus size (Harmon et al. 2010) translates into a $20 \%$ uncertainty on the flux and therefore restricts $\gamma$ to the range $0.8-1.2$. In 
Table 4. Results for the fits of the synthetic thermal light curve to the MIPS data using the HST and radar shape models.

\begin{tabular}{|c|c|c|c|c|c|c|c|c|c|}
\hline \multirow[t]{3}{*}{$\eta$} & \multirow[t]{3}{*}{$I$} & \multicolumn{4}{|c|}{ HST shape model } & \multicolumn{4}{|c|}{ Radar shape model } \\
\hline & & \multicolumn{2}{|c|}{ Prograde } & \multicolumn{2}{|c|}{ Retrograde } & \multicolumn{2}{|c|}{ Prograde } & \multicolumn{2}{|c|}{ Retrograde } \\
\hline & & $\gamma$ & $\chi^{2}$ & $\gamma$ & $\chi^{2}$ & $\gamma$ & $x^{2}$ & $\gamma$ & $\chi^{2}$ \\
\hline 0.7 & 0 & 0.83 & 37.0 & 0.83 & 37.6 & 0.91 & 193.2 & 0.90 & 190.7 \\
\hline 0.7 & 50 & 0.89 & 31.6 & 0.97 & 35.1 & 0.97 & 171.6 & 1.05 & 187.8 \\
\hline 0.7 & 100 & 0.97 & 27.8 & 1.07 & 30.6 & 1.04 & 155.1 & 1.14 & 161.7 \\
\hline 0.7 & 200 & 1.09 & 21.3 & 1.21 & 26.5 & 1.15 & 132.1 & 1.26 & 125.4 \\
\hline 0.7 & 400 & 1.23 & 19.4 & 1.33 & 26.4 & 1.28 & 114.6 & 1.37 & 108.5 \\
\hline 0.7 & 800 & 1.35 & 25.1 & 1.41 & 31.3 & 1.37 & 110.8 & 1.44 & 112.6 \\
\hline 0.8 & 0 & 0.90 & 36.9 & 0.90 & 37.6 & 0.99 & 194.1 & 0.99 & 191.6 \\
\hline 0.8 & 50 & 0.97 & 31.8 & 1.06 & 35.5 & 1.06 & 173.1 & 1.14 & 190.0 \\
\hline 0.8 & 100 & 1.05 & 28.1 & 1.17 & 30.8 & 1.14 & 156.5 & 1.25 & 164.8 \\
\hline 0.8 & 200 & 1.19 & 21.4 & 1.33 & 26.7 & 1.26 & 133.1 & 1.38 & 127.1 \\
\hline 0.8 & 400 & 1.36 & 19.4 & 1.47 & 26.7 & 1.40 & 115.1 & 1.50 & 108.8 \\
\hline 0.8 & 800 & 1.49 & 25.6 & 1.56 & 31.9 & 1.51 & 110.6 & 1.58 & 112.3 \\
\hline 0.9 & 0 & 0.97 & 37.1 & 0.97 & 37.8 & 1.07 & 194.9 & 1.06 & 192.4 \\
\hline 0.9 & 50 & 1.05 & 31.9 & 1.14 & 35.9 & 1.14 & 174.4 & 1.23 & 192.0 \\
\hline 0.9 & 100 & 1.14 & 28.2 & 1.27 & 31.1 & 1.23 & 157.9 & 1.35 & 167.4 \\
\hline 0.9 & 200 & 1.29 & 21.5 & 1.44 & 26.9 & 1.36 & 134.3 & 1.49 & 128.8 \\
\hline 0.9 & 400 & 1.48 & 19.5 & 1.60 & 27.1 & 1.52 & 115.7 & 1.63 & 109.3 \\
\hline 0.9 & 800 & 1.63 & 26.2 & 1.71 & 32.4 & 1.64 & 110.4 & 1.72 & 112.2 \\
\hline 1.0 & 0 & 1.04 & 37.2 & 1.05 & 37.8 & 1.14 & 195.7 & 1.14 & 193.0 \\
\hline 1.0 & 50 & 1.13 & 32.2 & 1.23 & 36.1 & 1.23 & 175.7 & 1.32 & 193.7 \\
\hline 1.0 & 100 & 1.22 & 28.5 & 1.37 & 31.5 & 1.32 & 159.2 & 1.45 & 169.9 \\
\hline 1.0 & 200 & 1.39 & 21.6 & 1.55 & 27.1 & 1.46 & 135.3 & 1.61 & 130.5 \\
\hline 1.0 & 400 & 1.59 & 19.5 & 1.73 & 27.3 & 1.64 & 116.2 & 1.76 & 109.8 \\
\hline 1.0 & 800 & 1.76 & 26.6 & 1.86 & 32.9 & 1.77 & 110.3 & 1.87 & 112.1 \\
\hline
\end{tabular}

Notes. $\eta$ is the beaming factor. $I$ is the nucleus thermal inertia $\left(\mathrm{J} \mathrm{K}^{-1} \mathrm{~m}^{-2} \mathrm{~s}^{-1 / 2}\right) \cdot \gamma$ is the derived scaling factor for the nucleus shape model to match the MIPS infrared flux. $\chi^{2}$ is the chi-square value; for reference, our model has 16 degrees of freedom (19 data points and 3 free parameters).

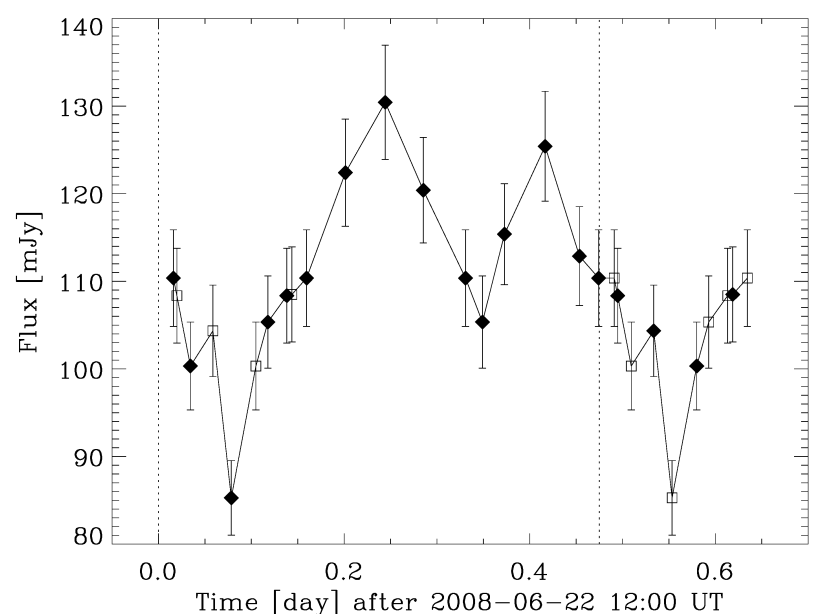

Fig. 4. MIPS thermal light curve at $24 \mu \mathrm{m}$. Filled diamonds correspond to the MIPS nucleus fluxes of Table 2. Square symbols have been phasefolded using a rotation period of $11.4 \mathrm{~h}$ (marked by the vertical dashed line at 0 day and 0.475 day). The light curve is double peaked, and one minimum (at 0.08 day) is stronger than the other (at 0.35 day).

Table 4, all the solutions with $\gamma>1.2$ can thus be discarded for the radar shape model. Conversely, the scale of the HST shape model is free, and all combinations $(\eta, I)$ are possible. Nevertheless, this scaling difference is not sufficient to explain the discrepancy between the two shape models. Even when $\gamma$ for the radar shape model is in the range $0.8-1.2$, as in Fig. $5(\eta=0.7$, $I=50 \mathrm{~J} \mathrm{~K}^{-1} \mathrm{~m}^{-2} \mathrm{~s}^{-1 / 2}$ ), the fit is still worse than with the HST shape model.

The first discrepancy is that the amplitude of the thermal light curve for the radar shape model is larger than that of the HST shape model, which is caused by the higher semi-major axis ratio of 1.7 for the radar shape model; the ratio is 1.4 for the HST shape model. The amplitude of the thermal light curve for the radar shape model is too large to properly fit the data.

The second discrepancy is minimum B, which is too deep for the radar shape model (Fig. 5). For the two shape models, minima B and D correspond to the scenario in which one lobe eclipses the other (Fig. 3). For the radar shape model, the eclipse is partial for the two minima, and because the illuminated cross-sections are close, the two minima are identical. For the HST shape model, minimum B corresponds to the scenario in which the larger body fully eclipses the other, and minimum D corresponds to the scenario in which the smaller body partially eclipses the other. In this case, minimum D is more pronounced than B as a result of projected shadows that lie close to the subsolar region from which the thermal flux is mainly emitted.

The different pole orientation of the HST and radar shape models partially explains these discrepancies because the projected shadows are different, but this is not sufficient, however. As shown in Fig. 6, even the radar shape model with the pole orientation of the HST shape model does not fit the data. This means that overall, the discrepancy between the HST and radar 


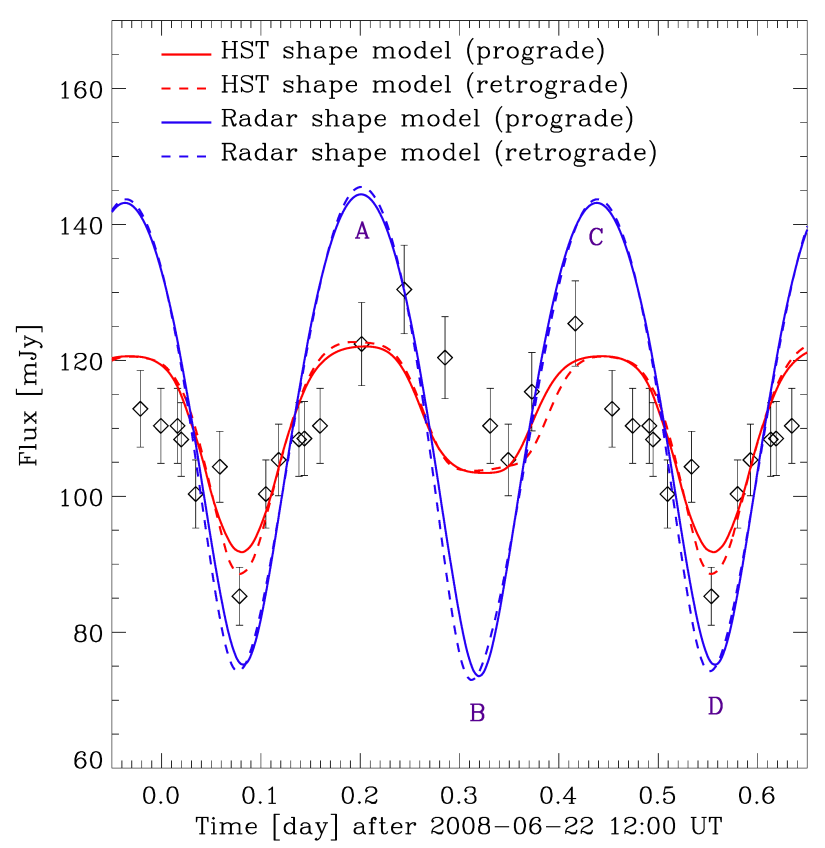

Fig. 5. Synthetic thermal light curves corresponding to the HST and radar shape models (prograde and retrograde) for the combination $\eta=0.7$ and $I=50 \mathrm{~J} \mathrm{~K}^{-1} \mathrm{~m}^{-2} \mathrm{~s}^{-1 / 2}$. The thermal light curves and the MIPS data have been extended beyond one rotation period for clarity. Letters A, B, C, and D correspond to the extrema and are connected to Fig. 3.

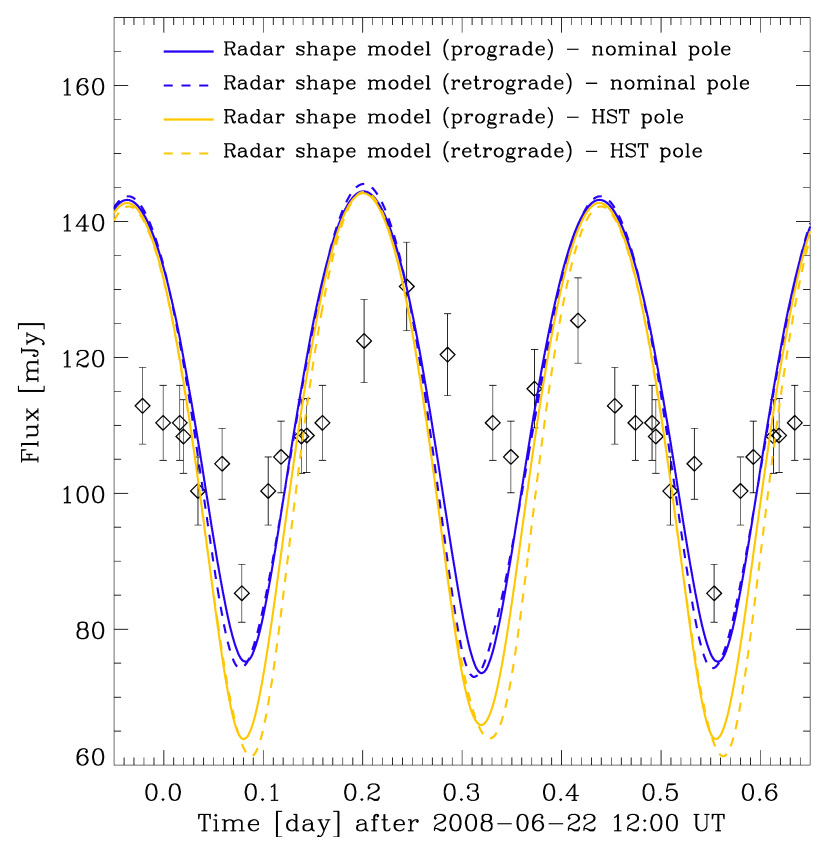

Fig. 6. Synthetic thermal light curves corresponding to the radar shape models (prograde and retrograde) for the combination $\eta=0.7$ and $I=50 \mathrm{~J} \mathrm{~K}^{-1} \mathrm{~m}^{-2} \mathrm{~s}^{-1 / 2}$, and two different pole solutions (nominal radar pole and HST pole).

solutions is a combination of their different pole orientation and shape.

From Table 4, the minimum $\chi^{2}$ value of 19.4 is obtained with the HST shape model for $\eta=0.7$ or $0.8, I=400 \mathrm{~J} \mathrm{~K}^{-1} \mathrm{~m}^{-2} \mathrm{~s}^{-1 / 2}$, prograde rotation. Our model has 16 degrees of freedom (19 data points and 3 free parameters), which gives a reduced chi-square of 1.2 , that is, a reasonable value close to 1 . We computed a $\Delta \chi^{2}$ of 36.2 for a confidence level of $99.7 \%$ (i.e., $3 \sigma$ for the normal distribution). At this confidence level, all the solutions with $\chi^{2}>$ $55.6(=19.4+36.2)$ can be rejected, which discards the radar shape model when we strictly follow this statistical criterion.

To conclude, the radar shape model has two identical minima and a large amplitude, which disagrees with the MIPS thermal light curve and the HST visible light curve (Lamy et al. 2008a). Moreover, the HST shape model always provides a better qualitative and quantitative fit to the data than the radar shape model, whatever the combination of $\eta$ and $I$, and regardless of whether the rotation is prograde or retrograde. We therefore favor the HST shape model over the radar shape model.

From Table 4, the $\chi^{2}$ value decreases when thermal inertia increases for the HST model by up to $I=400 \mathrm{~J} \mathrm{~K}^{-1} \mathrm{~m}^{-2} \mathrm{~s}^{-1 / 2}$. This provides the best fit to the data. The improvement of a factor $\approx 2$ of the chi-square value is not significant enough to reject any values of $\eta$ or $I$ at this stage, however, in particular because extrema $\mathrm{C}$ and $\mathrm{D}$ are not well reproduced by the highest values of thermal inertia (Fig. 7); additional constraints derived from IRS spectra are required.

\subsection{Dust $\epsilon$ f $\rho$ quantity}

We computed the quantity $\epsilon f \rho$ from all the MIPS images. This quantity, discussed in Kelley et al. (2013), is used to estimate the dust production in the infrared wavelength range by analogy with the $A f \rho$ quantity defined by A'Hearn et al. (1984) at visible wavelengths. The $\epsilon f \rho$ and $A f \rho$ quantities are independent of the aperture size $\rho$ if the coma has a canonical $1 / \rho$ radial brightness profile.

In our case, we computed the quantity $\epsilon f \rho$ with a dust temperature of $258 \mathrm{~K}$ derived from the IRS spectra (Sect. 5.4). We computed $\epsilon f \rho$ for different aperture sizes and found that its value varies by less than $5 \%$ for apertures between 10 and 30 pixels, consistent with a $1 / \rho$ coma radial brightness profile over this aperture range (Fig. 2). The $\epsilon f \rho$ values are reported in Table 2 .

The $\epsilon f \rho$ quantity is very stable over time and only varies between $322 \mathrm{~cm}$ and $329 \mathrm{~cm}$ during the $15 \mathrm{~h}$ of MIPS observations. The mean value is $\epsilon f \rho=325 \pm 36 \mathrm{~cm}$. The uncertainty mainly comes from the uncertainty on the dust temperature $(258 \pm 10 \mathrm{~K})$.

\section{IRS data analysis}

\subsection{Adjusting the model to the data}

The IRS spectrum was acquired on 2 November 2008 around 18:15 UT, in less than $10 \mathrm{~min}$. Unfortunately, the rotation period of the nucleus is not known with sufficient accuracy to rephase the spectrum with the HST or MIPS light curves, which were taken 38 and 233 days later, respectively. As a consequence, we adopted a conservative approach and assumed two extreme solutions for the cross-section viewed by SST at the time of observation, corresponding to the minimum and maximum cross-sections. As found in the previous section, only the HST shape model reasonably fits the MIPS light curve, therefore we performed the analysis with this shape model.

As explained in Sect. 3, five parameters need to be constrained by the spectrum: $\gamma, \eta$, and $I$ for the nucleus, and $\mathcal{A}$ and $T_{\text {dust }}$ for the dust coma. There are more parameters than constraints, and several solutions are possible. However, for a given combination of $\eta$ and $I$ that defines the shape of the nucleus SED, we can determine $T_{\text {dust }}$ that controls the shape of the dust coma SED. The scaling factors $\gamma$ and $\mathcal{A}$ can then be adjusted to match 

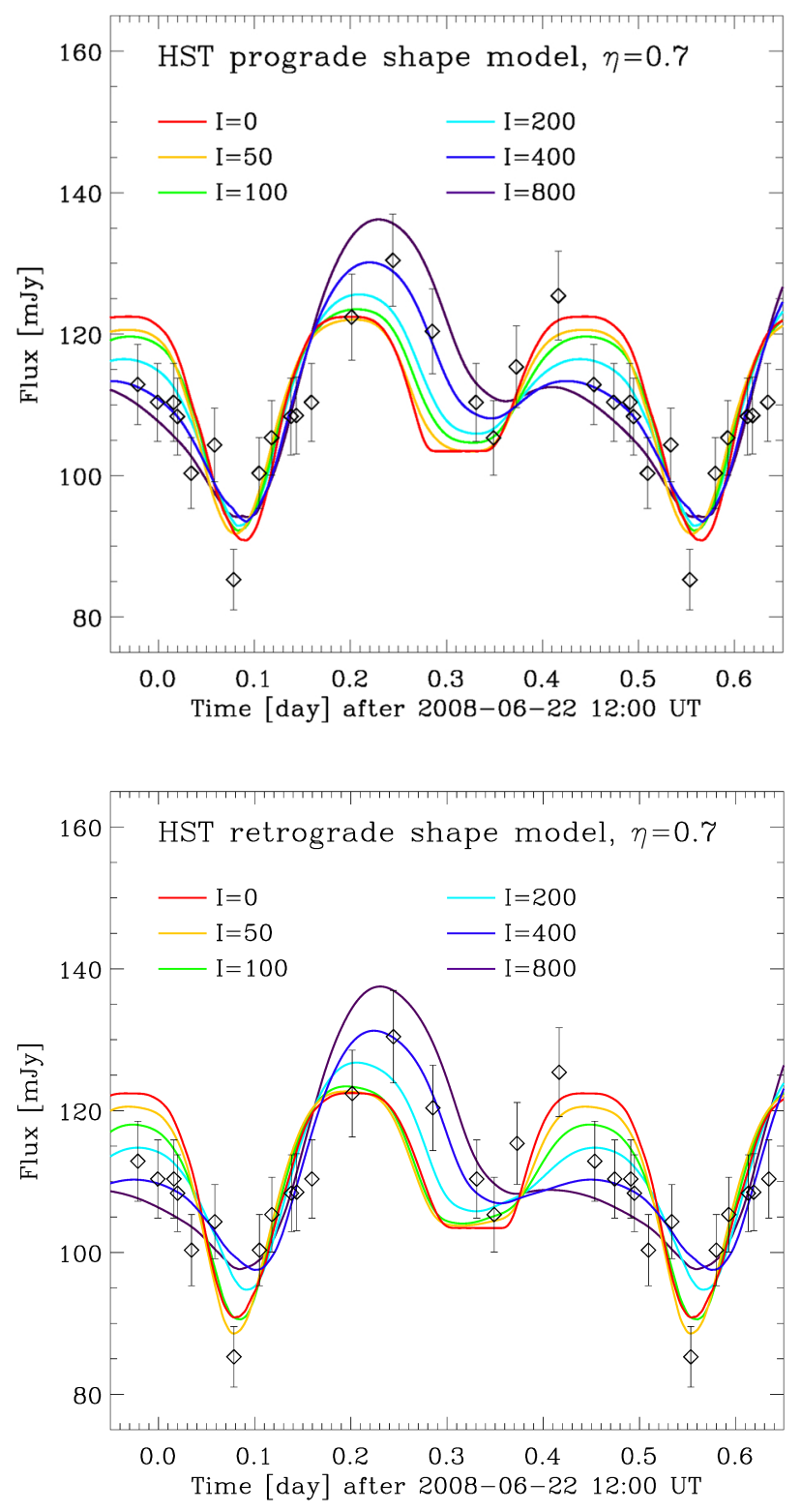

Fig. 7. Synthetic thermal light curves using the HST shape model (prograde at the top, retrograde at the bottom) for $\eta=0.7$ and different values of thermal inertia $I$ between 0 and $800 \mathrm{~J} \mathrm{~K}^{-1} \mathrm{~m}^{-2} \mathrm{~s}^{-1 / 2}$.

the data. As a result, for each combination of $\eta$ in the range $0.7-$ 1.0 and $I$ in the range $0-800 \mathrm{~J} \mathrm{~K}^{-1} \mathrm{~m}^{-2} \mathrm{~s}^{-1 / 2}$, we searched for the values of $T_{\text {dust }}, \gamma$, and $\mathcal{A}$ that minimize the chi-square value between the synthetic SED $\left(F_{\text {nucl }}+F_{\text {coma }}\right)$ and the data. Because the SL and LL modes have different slit widths (3.6-3.7" vs. $\left.10.5-10.7^{\prime \prime}\right)$, there is one $\mathcal{A}$ value for each of them $\left(\mathcal{A}_{\mathrm{SL}}\right.$ and $\mathcal{A}_{\mathrm{LL}}$ ). We estimated the uncertainty to $10 \%$ on $\gamma$ (IRS), $5 \mathrm{~K}$ on $T_{\text {dust }}, 3 \times 10^{6} \mathrm{~m}^{2}$ on $\mathcal{A}_{\mathrm{SL}}$, and $5 \times 10^{6} \mathrm{~m}^{2}$ on $\mathcal{A}_{\mathrm{LL}}$, in order to keep the residuals within the $1 \sigma$ error bars. Results are given in Table 5 and illustrated in Fig. 8.

\subsection{Roughness and thermal inertia}

The shape model did not change between the IRS and MIPS observations, therefore the scaling factors $\gamma$ are expected to be compatible with both IRS and MIPS data, that is, the value for $\gamma$ (MIPS) must be between the two values of $\gamma$ (IRS) that correspond to the minimum and maximum cross-section, within the error bars. The solutions in bold in Table 5 satisfy this criterion. They correspond to $\eta=0.7$ with $I$ in the range $0-100 \mathrm{~J} \mathrm{~K}^{-1} \mathrm{~m}^{-2} \mathrm{~s}^{-1 / 2}$, and to $\eta=0.8$ with a null thermal inertia. Strictly speaking, two additional solutions $(\eta=0.7$; $\left.I=200 \mathrm{~J} \mathrm{~K}^{-1} \mathrm{~m}^{-2} \mathrm{~s}^{-1 / 2}\right)$ and $\left(\eta=0.8 ; I=50 \mathrm{~J} \mathrm{~K}^{-1} \mathrm{~m}^{-2} \mathrm{~s}^{-1 / 2}\right)$ are also possible, but are marginally compatible with the data because they imply that we observed exactly at the maximum cross-section, moreover, at the $1 \sigma$ lower limit for $\gamma$ (IRS); we therefore rejected them. For other values of $\eta$ and $I$, the difference between the scaling factor $\gamma$ (IRS) and $\gamma$ (MIPS) is too large or the fit is unphysical (e.g., $\mathcal{A}_{\mathrm{SL}}=0$ with no coma in the SL mode); we did not list all these solutions in Table 5.

The value of $0.7-0.8$ for the beaming factor is low, probably indicating a high surface roughness. The thermal inertia of the nucleus is in the range of $0-100 \mathrm{~J} \mathrm{~K}^{-1} \mathrm{~m}^{-2} \mathrm{~s}^{-1 / 2}$, which is compatible with thermal inertia values derived for other comets, for example, $<45 \mathrm{~J} \mathrm{~K}^{-1} \mathrm{~m}^{-2} \mathrm{~s}^{-1 / 2}$ (Groussin et al. 2013) and $<200 \mathrm{~J} \mathrm{~K}^{-1} \mathrm{~m}^{-2} \mathrm{~s}^{-1 / 2}$ (Davidsson et al. 2013) for comet 9P/Tempel 1, <200 J K ${ }^{-1} \mathrm{~m}^{-2} \mathrm{~s}^{-1 / 2}$ for comet 103P/Hartley 2 (Groussin et al. 2013), or 10-30 J K ${ }^{-1} \mathrm{~m}^{-2} \mathrm{~s}^{-1 / 2}$ (Schloerb et al. 2015) and 0-350 J K ${ }^{-1} \mathrm{~m}^{-2} \mathrm{~s}^{-1 / 2}$ for comet 67P/ChuryumovGerasimenko (Marshall et al. 2018). As suggested by Boissier et al. (2011), who derived a value $\leq 10 \mathrm{~J} \mathrm{~K}^{-1} \mathrm{~m}^{-2} \mathrm{~s}^{-1 / 2}$ for $8 \mathrm{P} /$ Tuttle from millimeter observations, the lowest values are probably better.

\subsection{Nucleus size and geometric albedo}

The scaling factor for IRS depends on the visible cross-section at the time of observation, which is unknown. As a consequence, the scaling factor derived from the MIPS thermal light curve is more robust, and we chose its value to determine the size. For the valid combinations of $\eta$ and $I$ discussed above, the scaling factor lies in the range $0.83-0.97$ or $0.90 \pm 0.07$. Adding quadratically a flux calibration uncertainty of $5 \%$ yields $\gamma=0.90 \pm 0.09$. Applying this result to the size of the two contact spheres yields radii of $2.7 \pm 0.1 \mathrm{~km}$ and $1.1 \pm 0.1 \mathrm{~km}$. For reference, a sphere with an "equivalent" radius of $2.9 \mathrm{~km}$ would have the same maximum cross-section.

Lamy et al. (2008a) derived an apparent $R$ magnitude of $15.7 \pm 0.2$ with the HST on 10 December $2007\left(r_{\mathrm{h}}=1.26 \mathrm{AU}\right.$, $\Delta=0.49 \mathrm{AU}$ and $\alpha=46^{\circ}$ ), which corresponds to the mean value of the visible light curve. From this magnitude, we derived a geometric albedo of $0.042 \pm 0.008$ in the $R$ band, using the above equivalent radius of $2.9 \mathrm{~km}$ and a linear phase correction with a phase coefficient $\beta=0.04 \mathrm{mag} \mathrm{deg}^{-1}$.

\subsection{Dust color temperature and $\epsilon f \rho$ quantity}

For possible solutions $(\eta, I)$, the dust temperature is well constrained to $258 \pm 10 \mathrm{~K}$. This is $37 \mathrm{~K}$ higher than the temperature of an isothermal low-albedo dust grain in thermal equilibrium at $r=1.6 \mathrm{AU}$ from the Sun $(T \approx 221 \mathrm{~K})$. This indicates that dust grains that contribute to the thermal infrared flux have a typical size of $\approx 10 \mu \mathrm{m}$ (diameter) according to Gicquel et al. (2012), assuming porous amorphous carbon dust grains with a fractal dimension of 2.727 for the porosity model. We emphasize that this only provides a rough estimate of the grain size because cometary dust grains are complex in terms of physical properties and composition (Wooden et al. 2017).

From the dust cross-section in SL and LL mode, we can derive the $\epsilon f \rho$ quantity (Sect. 4.3). In our case, the filling factor $f$ is equal to $\mathcal{A}_{\mathrm{SL}}$ for the SL mode or $\mathcal{A}_{\mathrm{LL}}$ for the LL mode, divided by the field of view in $\mathrm{m}^{2}$. To roughly estimate the 
Table 5. Results for the fit of the synthetic SED to the IRS data using the HST shape model.

\begin{tabular}{|c|c|c|c|c|c|c|c|c|}
\hline$\eta$ & $\begin{array}{c}I \\
{\left[\mathrm{~J} \mathrm{~K}^{-1} \mathrm{~m}^{-2} \mathrm{~s}^{-1 / 2}\right]}\end{array}$ & Rotation & Cross-section & $\gamma(\mathrm{IRS})$ & $\begin{array}{c}T_{\text {dust }} \\
{[\mathrm{K}]}\end{array}$ & $\begin{array}{c}\mathcal{A}_{\mathrm{SL}} \\
{\left[10^{6} \mathrm{~m}^{2}\right]}\end{array}$ & $\begin{array}{c}\mathcal{A}_{\mathrm{LL}} \\
{\left[10^{6} \mathrm{~m}^{2}\right]}\end{array}$ & $\gamma(\mathrm{MIPS})$ \\
\hline \multirow{2}{*}{0.7} & \multirow{2}{*}{$\mathbf{0}$} & \multirow{2}{*}{ N/A } & $\min$ & 1.31 & 262 & 24 & 75 & \multirow{2}{*}{0.83} \\
\hline & & & $\max$ & 0.64 & 267 & 29 & 79 & \\
\hline \multirow{4}{*}{0.7} & \multirow{4}{*}{50} & \multirow{2}{*}{ Prograde } & $\min$ & 1.61 & 254 & 13 & 64 & \multirow{2}{*}{0.89} \\
\hline & & & $\max$ & 0.75 & 264 & 25 & 75 & \\
\hline & & \multirow{2}{*}{ Retrograde } & $\min$ & 2.25 & 267 & 14 & 57 & \multirow{2}{*}{0.97} \\
\hline & & & $\max$ & 0.95 & 265 & 24 & 73 & \\
\hline \multirow{4}{*}{0.7} & \multirow{4}{*}{100} & \multirow{2}{*}{ Prograde } & $\min$ & 1.79 & 248 & 7 & 60 & \multirow{2}{*}{0.97} \\
\hline & & & $\max$ & 0.90 & 258 & 21 & 73 & \\
\hline & & \multirow{2}{*}{ Retrograde } & $\min$ & 3.42 & 270 & 0 & 34 & \multirow{2}{*}{1.07} \\
\hline & & & $\max$ & 1.37 & 262 & 16 & 63 & \\
\hline \multirow{4}{*}{0.7} & \multirow{4}{*}{200} & \multirow{2}{*}{ Prograde } & $\min$ & 2.12 & 242 & 0 & 53 & \multirow{2}{*}{1.09} \\
\hline & & & $\max$ & 1.21 & 249 & 12 & 67 & \\
\hline & & \multirow{2}{*}{ Retrograde } & $\min$ & 0.47 & 302 & 31 & 70 & \multirow{2}{*}{1.21} \\
\hline & & & $\max$ & 2.21 & 251 & 0 & 44 & \\
\hline \multirow{2}{*}{0.8} & \multirow{2}{*}{$\mathbf{0}$} & \multirow{2}{*}{ N/A } & $\min$ & 1.85 & 255 & 17 & 69 & \multirow{2}{*}{0.90} \\
\hline & & & $\max$ & 0.95 & 259 & 23 & 75 & \\
\hline \multirow{4}{*}{0.8} & \multirow{4}{*}{50} & \multirow{2}{*}{ Prograde } & $\min$ & 2.21 & 246 & 2 & 54 & \multirow{2}{*}{0.97} \\
\hline & & & $\max$ & 1.09 & 255 & 18 & 70 & \\
\hline & & \multirow{2}{*}{ Retrograde } & $\min$ & 3.22 & 262 & 3 & 44 & \multirow{2}{*}{1.06} \\
\hline & & & $\max$ & 1.33 & 260 & 17 & 66 & \\
\hline 09 & 0 & N/A & $\min$ & 2.51 & 247 & 7 & 61 & 097 \\
\hline 0.9 & 0 & IV/A & $\max$ & 1.28 & 253 & 16 & 69 & 0.91 \\
\hline 10 & 0 & $\mathrm{~N} / \mathrm{A}$ & $\min$ & 3.08 & 242 & 0 & 54 & 104 \\
\hline & & & $\max$ & 1.67 & 245 & 7 & 63 & \\
\hline
\end{tabular}

Notes. Combinations $(\eta, I)$ in bold are compatible with both IRS and MIPS observations. Rotation defines the sense of rotation (P for prograde, $\mathrm{R}$ for retrograde, N/A for a null thermal inertia). Cross-section is the cross-section facing SST at the time of observation: minimum or maximum (see text for details). $\gamma$ (IRS) is the derived scaling factor for the IRS infrared flux. $T_{\text {dust }}$ is the derived temperature of the dust. $\mathcal{A}_{\mathrm{SL}}$ is the derived dust cross-section in the SL field of view. $\mathcal{A}_{\mathrm{LL}}$ is the derived dust cross-section in the LL field of view. $\gamma$ (MIPS) is the scaling factor for the MIPS infrared flux from Table 4.

value of $\mathcal{A}_{\mathrm{SL}}$ and $\mathcal{A}_{\mathrm{LL}}$ at the time of observation, that is, when $\gamma$ (IRS) equals $\gamma$ (MIPS) in Table 5, we interpolated linearly between the values at minimum and maximum cross-sections. For the possible solutions $(\eta, I)$, we obtain $\mathcal{A}_{\mathrm{SL}}$ in the range 23-28 $\times 10^{6} \mathrm{~m}^{2}$ and $\mathcal{A}_{\mathrm{LL}}$ in the range $72-78 \times 10^{6} \mathrm{~m}^{2}$. The fields of view being $1.9 \times 10^{13} \mathrm{~m}^{2}$ in the SL mode $\left(2.6^{\prime \prime}\right.$ equivalent radius) and $1.7 \times 10^{14} \mathrm{~m}^{2}$ in the $\mathrm{LL}$ mode $\left(7.8^{\prime \prime}\right.$ equivalent radius), $\rho$ is equal to $2450 \mathrm{~km}$ and $7440 \mathrm{~km}$, respectively. Overall, we obtained $\epsilon f \rho=314 \pm 31 \mathrm{~cm}$ in the SL mode and $\epsilon f \rho=$ $305 \pm 13 \mathrm{~cm}$ in the LL mode. Remarkably, the two values are well consistent with each other and correspond to a mean value $\epsilon f \rho=310 \pm 34 \mathrm{~cm}$.

This $\epsilon f \rho$ value is similar to the value of $325 \pm 36 \mathrm{~cm}$ derived from the MIPS images (Sect. 4.3), indicating that the dust production did not change significantly between the IRS observations at $r_{\mathrm{h}}=1.6 \mathrm{AU}$ pre-perihelion and the MIPS observations at $r_{\mathrm{h}}=2.2 \mathrm{AU}$ post-perihelion. The $\epsilon f \rho$ value is also higher than the Af $\rho$ values derived in the visible during the same perihelion passage (Sect. 1), which could indicate more dust particles with sizes of $\approx 10 \mu \mathrm{m}$ size than submicron size. Finally, the $\epsilon f \rho$ values of comet $8 \mathrm{P} /$ Tuttle are comparable to values obtained for other comets (Kelley et al. 2013).

\subsection{Water production rate}

The IRS spectrum allowed detecting the $v_{2}$ water band around $6.5 \mu \mathrm{m}$, as illustrated in Fig. 9. The strongest water emission features are all at their expected wavelength (Bockelée-Morvan et al. 2009). We modeled the continuum with a linear function, which is a good approximation over the small wavelength range of $5.5-7.3 \mu \mathrm{m}$. The intensity of the $v_{2}$ water band, integrated over the wavelength range 5.8-6.9 $\mu \mathrm{m}$, amounts to $5.6 \pm 1.0 \times 10^{-20} \mathrm{~W} \mathrm{~cm}-2$ in an equivalent circular aperture of radius $2.85^{\prime \prime}$. Using a Haser model with a g-factor of $2.41 \times 10^{-4} \mathrm{~s}^{-1}$ at $r_{\mathrm{h}}=1$ AU (Bockelée-Morvan et al. 2009) and a typical gas velocity of $0.5 \mathrm{~km}^{-1}$ at $r_{\mathrm{h}}=1.6 \mathrm{AU}$, we derived a water production rate of $1.1 \pm 0.2 \times 10^{28}$ molecules $\mathrm{s}^{-1}$ or $340 \pm 60 \mathrm{~kg} \mathrm{~s}^{-1}$.

For a spherical nucleus with a radius of about $2.9 \mathrm{~km}$, this implies an active fraction of $\approx 9 \%$, derived from the water production rate of a spherical nucleus made of water ice only and located at the same heliocentric distance. This value is in agreement with the active fraction of $3-15 \%$ derived from the water production rates at perihelion, assuming a radius of $3 \mathrm{~km}$ (Sect. 1). The active fraction of 8P/Tuttle is comparable to that of 

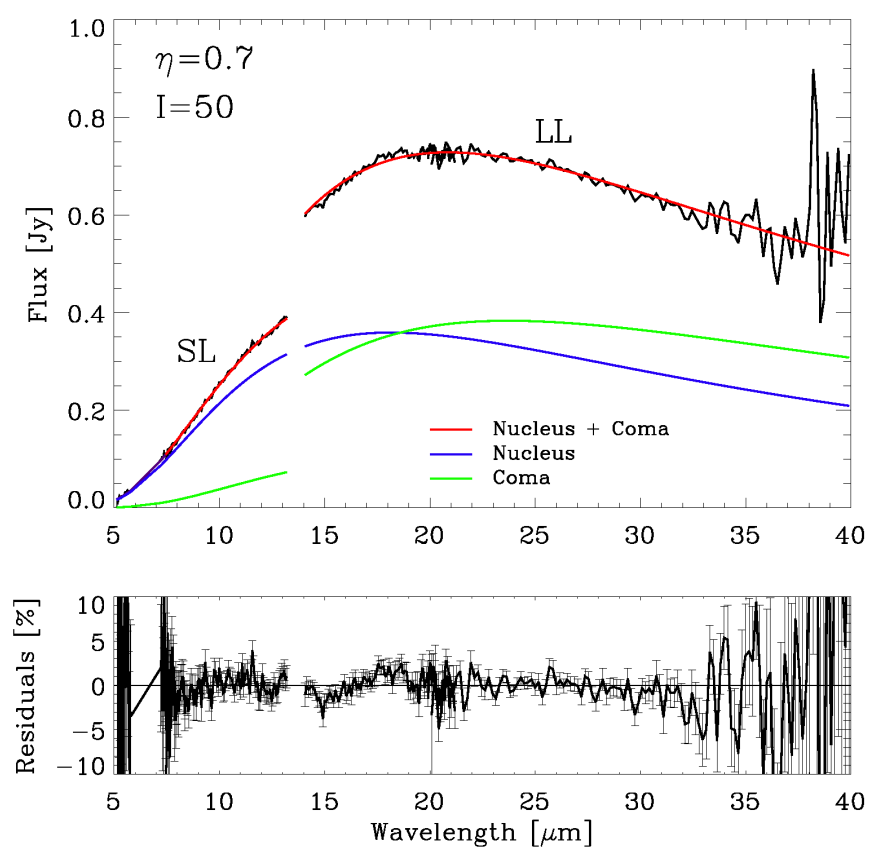

Fig. 8. IRS data and synthetic SED for the combination $\eta=0.7$ and $I=50 \mathrm{~J} \mathrm{~K}^{-1} \mathrm{~m}^{-2} \mathrm{~s}^{-1 / 2}$ (minimum cross-section). The blue line shows the contribution from the nucleus, the green line represents that from the dust coma, and the red line is the sum of both (nucleus + coma). Residuals correspond to the difference between the model (nucleus + dust coma) and the data. The discontinuity between the SL and LL mode results from their different field of views, with less coma in SL mode (smaller field of view) than in LL mode (larger field of view).

$\approx 10 \%$ for $1 \mathrm{P} /$ Halley (Keller et al. 1987), a comet from the same dynamical family.

\subsection{Dust mineralogy}

The mid-infrared spectral domain contains features that are diagnostic of surface composition (Wooden et al. 2017). The strongest emissions are expected from amorphous silicates at $\approx 8-12 \mu \mathrm{m}$ and forsterite $\approx 19.2-20.5 \mu \mathrm{m}$. Figure 10 shows the coma dust spectrum of comet $8 \mathrm{P} /$ Tuttle. This is the IRS spectrum from which we subtracted the nucleus synthetic spectrum (for the case $\eta=0.7, I=50 \mathrm{~J} \mathrm{~K}^{-1} \mathrm{~m}^{-2} \mathrm{~s}^{-1 / 2}$, prograde rotation, and minimum cross-section) and then divided by the corresponding synthetic coma spectrum $\left(T_{\text {dust }}=254 \mathrm{~K}\right)$; the spectrum is normalized to unity at $13.0 \mu \mathrm{m}$. Because we used a single temperature for the dust coma over the full 5-40 $\mu \mathrm{m}$ spectral range, the continuum is overestimated around $8 \mu \mathrm{m}$ (blue spectrum in Fig. 10). To correct for this effect and obtain a better spectrum over the full wavelength range, we used a slightly lower temperature $T_{\text {dust }}=248 \mathrm{~K}$ in the SL range (red spectrum in Fig. 10).

The coma dust spectrum seems to exhibit the broad amorphous silicate emission at $\approx 8-12 \mu \mathrm{m}$ ( $1.5 \sigma$ confidence level $)$, as has previously been observed on several comets (Fig. 11), despite the poor $\mathrm{S} / \mathrm{N}$ of the spectrum in this wavelength range. The spectrum also exhibits a second broad emission at $\approx 16-21 \mu \mathrm{m}(5 \sigma$ confidence level) and a large bump around $25 \mu \mathrm{m}$ ( $2 \sigma$ confidence level). The broad emissions around $10 \mu \mathrm{m}$ and $18 \mu \mathrm{m}$ are consistent with those of amorphous pyroxene, which exibits similar emissions at $\approx 9.0-11.0 \mu \mathrm{m}$ and $\approx 16.0-22.0 \mu \mathrm{m}$ (Wooden et al. 2005; Reach et al. 2010) (Fig. 11, lower panel). The $18 \mu \mathrm{m}$ emission is weak (only 6\%), which is consistent with our inferred
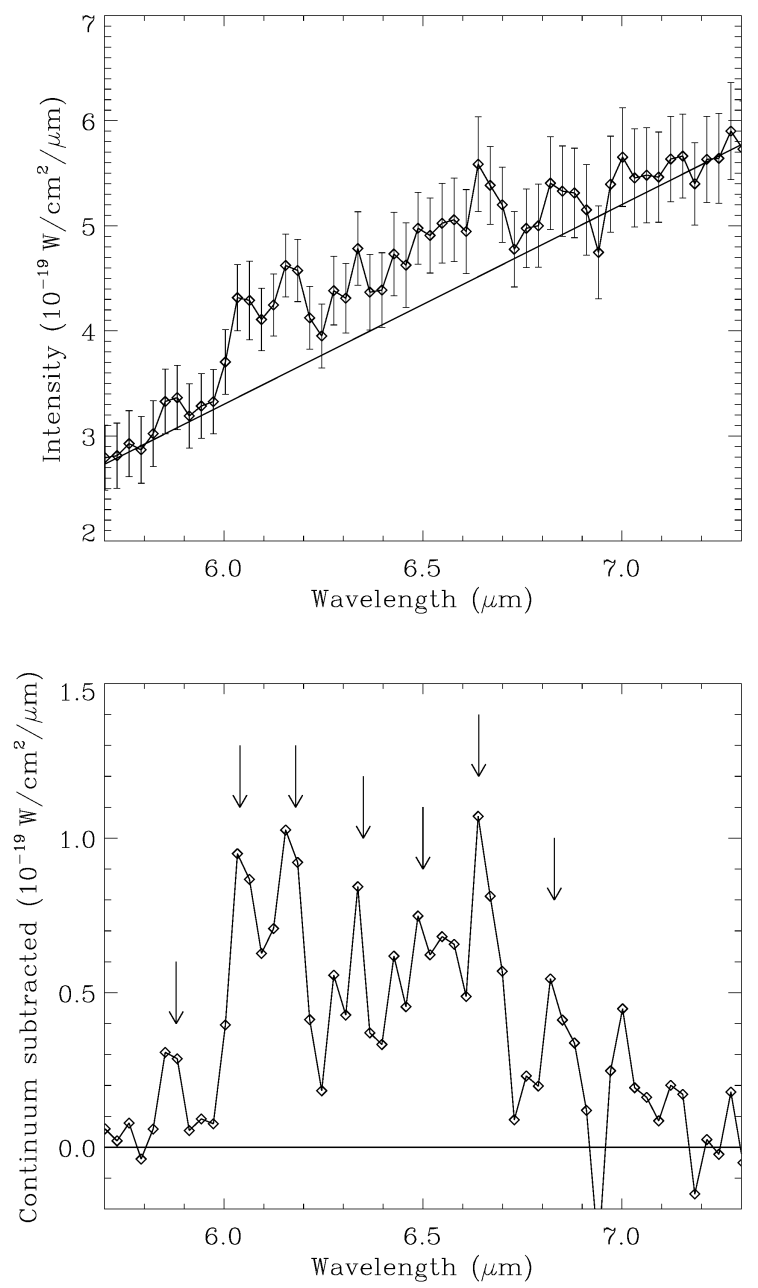

Fig. 9. Spectrum of the coma of $8 \mathrm{P} /$ Tuttle showing the $v_{2}$ water band around $6.5 \mu \mathrm{m}$. Upper panel: spectrum with the computed linear continuum. Lower panel: continuum-subtracted spectrum. The arrows indicate the location of the strongest $v_{2}$ water emission features.

grain size of $\approx 10 \mu \mathrm{m}$ (diameter) because large grains reduce the contrast of emission features compared to small micron to submicron size grains. Amorphous pyroxene was also detected on comet 17P/Holmes soon after its explosion in November 2007 (Reach et al. 2010). The large bump around $25 \mu \mathrm{m}$ is difficult to explain in terms of mineralogical composition, however.

In contrast to other comets (Fig. 11), the spectrum of 8P/Tuttle lacks the forsterite features at 19.2, 23.7, and $27.8 \mu \mathrm{m}$ (Wooden et al. 2017). Additionally, the large bump at $25 \mu \mathrm{m}$ is located in a region where other comets do not show any obvious emission features. These differences are puzzling and may result from the complexity of cometary dust grains in terms of physical properties (size distribution, shape, porosity, and temperature) and mineralogical composition.

\section{Conclusions}

We have presented infrared observations of comet 8P/Tuttle performed with the MIPS and IRS instruments of the Spitzer Space Telescope. Our main results are summarized in Table 6 and below.

1. The HST shape model outperforms the radar shape model, providing a better qualitative and quantitative fit to the MIPS thermal light curve. This fit leads to a bilobate shape 


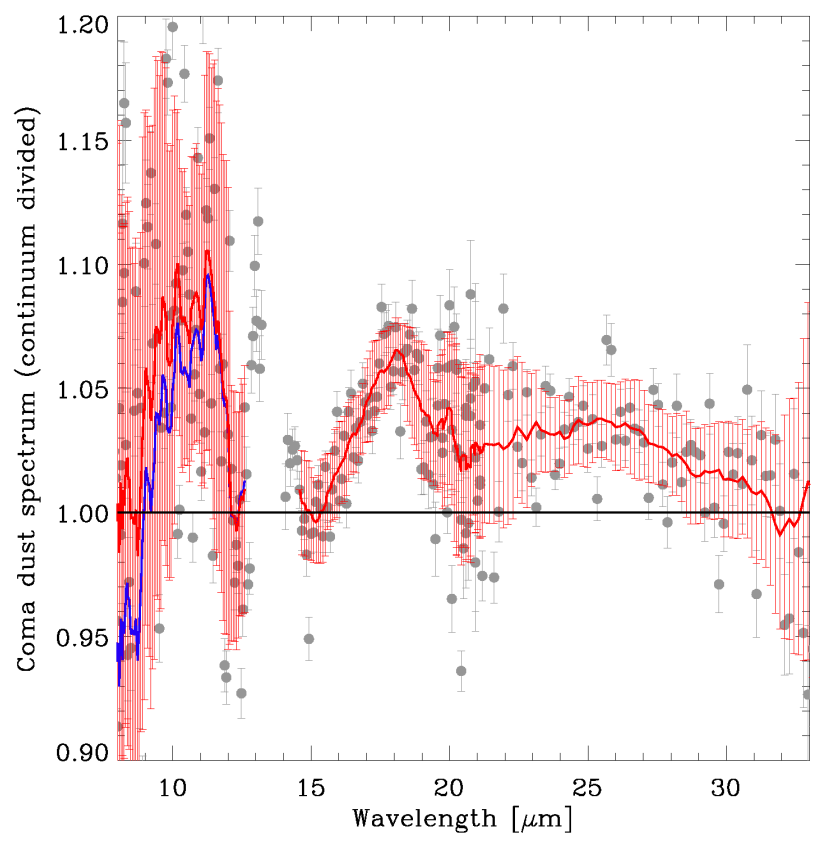

Fig. 10. Coma dust spectrum of comet $8 \mathrm{P} /$ Tuttle, continuum divided and normalized to unity at $13.0 \mu \mathrm{m}$. The red line corresponds to a smoothing over the data points, with a smoothing window of 15 data points. The red error bars correspond to the variance of the data points within the smoothing window. The blue line shows the spectrum in the SL mode assuming a single temperature for the dust coma, which causes the continuum to be overestimated around $8 \mu \mathrm{m}$ (see text for details).

composed of two spheres in contact with radii of $2.7 \pm 0.1 \mathrm{~km}$ and $1.1 \pm 0.1 \mathrm{~km}$ and a pole orientation given by $\mathrm{RA}=285 \pm 12^{\circ}$ and $\mathrm{Dec}=+20 \pm 5^{\circ}$.

2. The $R$-band geometric albedo is $0.042 \pm 0.008$.

3. The thermal inertia is in the range $0-100 \mathrm{~J} \mathrm{~K}^{-1} \mathrm{~m}^{-2} \mathrm{~s}^{-1 / 2}$; within this range, we favor the lowest values.

4. The surface roughness is rather high with a beaming factor in the range $0.7-0.8$.

5. The water production rate is estimated to be $1.1 \pm 0.2 \times 10^{28}$ molecules $\mathrm{s}^{-1}$ at $r_{\mathrm{h}}=1.6 \mathrm{AU}$ pre-perihelion, which corresponds to an active fraction of $\approx 9 \%$, similar to that of $1 \mathrm{P} / \mathrm{Halley}$, a comet of the same dynamical family.

6. The dust $\epsilon f \rho$ quantity amounts to $310 \pm 34 \mathrm{~cm}$ at $r_{\mathrm{h}}=1.6 \mathrm{AU}$ pre-perihelion, and to $325 \pm 36 \mathrm{~cm}$ at $r_{\mathrm{h}}=2.2 \mathrm{AU}$ postperihelion.

7. The dust grain temperature is estimated to $258 \pm 10 \mathrm{~K}$, which is $37 \mathrm{~K}$ higher than the thermal equilibrium temperature at $r_{\mathrm{h}}=1.6 \mathrm{AU}$. This indicates that the dust grains contributing to the thermal infrared flux have a typical size of $\approx 10 \mu \mathrm{m}$.

8. The dust spectrum exhibits broad emissions around $10 \mu \mathrm{m}$ ( $1.5 \sigma$ confidence level) and $18 \mu \mathrm{m}$ ( $5 \sigma$ confidence level) that we attribute to amorphous pyroxene.

Although $8 \mathrm{P} /$ Tuttle is a nearly isotropic comet, the above results do not indicate that its physical properties are intrinsically different from those of ecliptic comets. The size, albedo, thermal properties, and the water and dust production rate are not unusual compared with those of ECs (Lamy et al. 2004; BockeléeMorvan et al. 2004). These similarities between NIC and EC comets have previously been observed and suggest that they "formed in largely overlapping regions where the giant planets are today" (A'Hearn et al. 2012).

The discrepancy between the HST and radar shape models comes from their different shapes and pole orientations. The RA
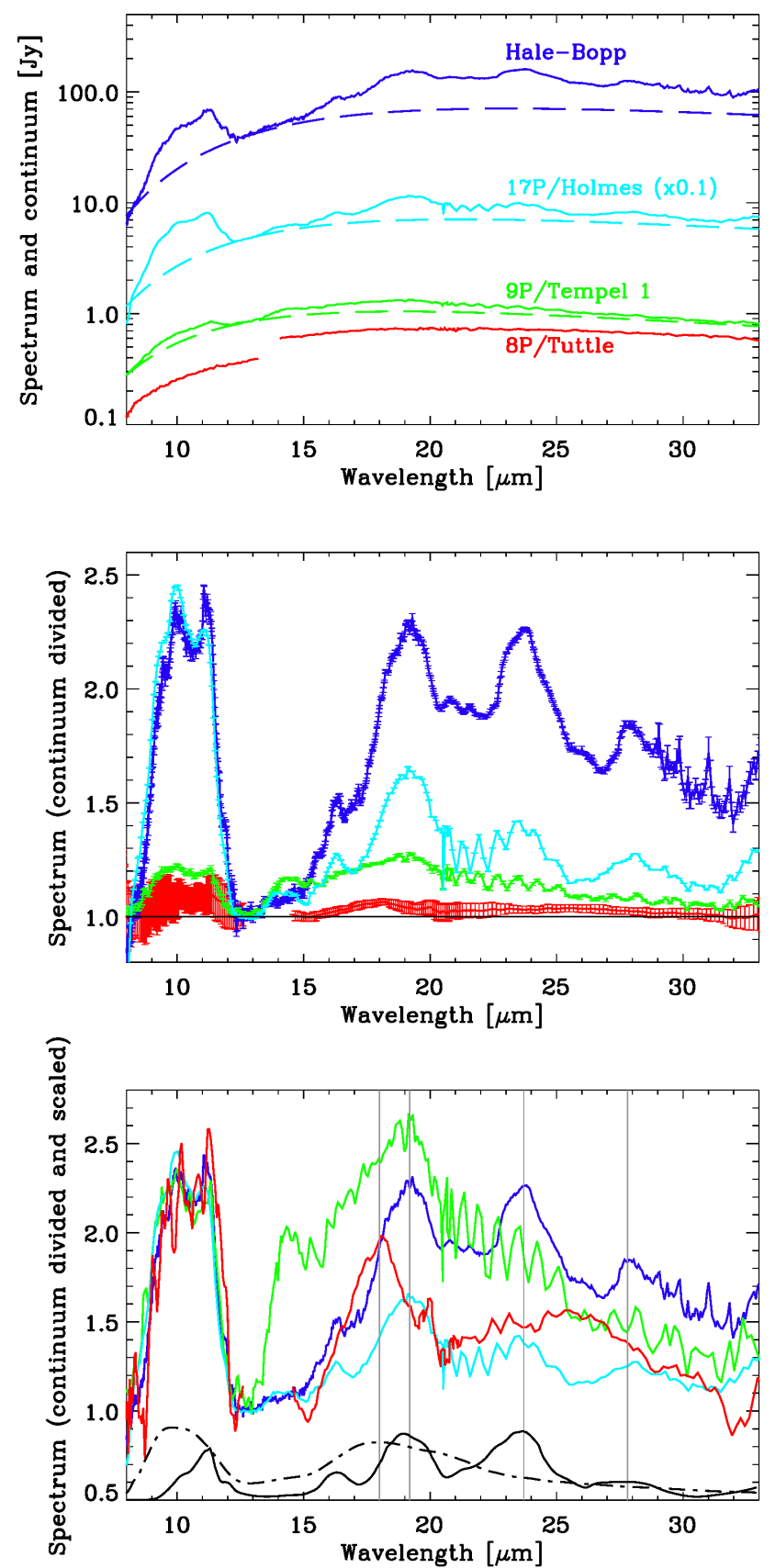

Fig. 11. Upper panel: spectra of several comets, for comparison with 8P/Tuttle. The data are from Crovisier et al. (1997) for Hale-Bopp, from Lisse et al. (2006) and Kelley \& Wooden (2009) for 9P/Tempel 1 (preDeep Impact), and from Reach et al. (2010) for 17P/Holmes (soon after the November 2007 explosion). The spectrum of 17P/Holmes has been divided by 10 for clarity. Dashed lines correspond to a blackbody continuum of $220 \mathrm{~K}$ for Hale-Bopp, $240 \mathrm{~K}$ for $17 \mathrm{P} / \mathrm{Holmes}$, and $270 \mathrm{~K}$ for $9 \mathrm{P} /$ Tempel 1 . The continuum for $8 \mathrm{P} /$ Tuttle is not shown for clarity, but it is identical to that of Fig. 8. Middle panel: continuum-divided spectra, using the same color code as in the upper panel. Error bars are indicated. For $8 \mathrm{P} /$ Tuttle, this is the same spectrum as in Fig. 10. The contrast of the spectrum of 8P/Tuttle is far lower than that of other comets. Lower panel: continuum-divided spectra, scaled to facilitate comparison, so that they all have the same value at $11 \mu \mathrm{m}$. Same color code as in the other panels. The black spectra at the bottom are those of crystalline forsterite (solid line) and amorphous pyroxene (dash-dotted line), extracted from Reach et al. (2010) for grains with a diameter of $2 \mu \mathrm{m}$. The vertical gray lines highlight the position of the amorphous pyroxene emission around $18 \mu \mathrm{m}$ observed on comet 8P/Tuttle compared with that of forsterite at 19.2, 23.7, and $27.8 \mu \mathrm{m}$ observed on the other comets. 
Table 6. Properties of comet 8P/Tuttle derived from our Spitzer Space Telescope observations.

\begin{tabular}{ll}
\hline \hline Nucleus & \\
Shape & Two spheres in contact (binary) \\
Size of each sphere & $2.7 \pm 0.1 \mathrm{~km}$ and $1.1 \pm 0.1 \mathrm{~km}$ (radii) \\
Pole orientation & $\mathrm{RA}=285 \pm 12^{\circ}$ and $\mathrm{Dec}=+20 \pm 5^{\circ}$ \\
Geometric albedo & $0.042 \pm 0.008(R$-band $)$ \\
\hline Roughness and thermal inertia \\
Valid combinations & $\begin{array}{l}\eta=0.7 \text { and } I=0-100 \mathrm{~J} \mathrm{~K}^{-1} \mathrm{~m}^{-2} \mathrm{~s}^{-1 / 2} \\
\text { or } \eta=0.8 \text { and } I=0 \mathrm{~J} \mathrm{~K} \mathrm{~K}^{-1} \mathrm{~m}^{-2} \mathrm{~s}^{-1 / 2}\end{array}$ \\
\hline Production rates & $1.1 \pm 0.2 \times 10^{28} \mathrm{~s}^{-1}(1.6 \mathrm{AU}$ pre-peri.) \\
Water $Q_{\mathrm{H}_{2} \mathrm{O}}$ & $\approx 9 \%$ \\
Active fraction & $310 \pm 34 \mathrm{~cm}(1.6 \mathrm{AU}$ pre-perihelion $)$ \\
Dust $\epsilon f \rho$ & $325 \pm 36 \mathrm{~cm}(2.2 \mathrm{AU}$ post-perihelion) \\
& \\
\hline Dust properties & $258 \pm 10 \mathrm{~K}(1.6 \mathrm{AU}$ pre-perihelion) \\
$T_{\text {dust }}$ & $\approx 10 \mu \mathrm{m}($ diameter $)$ \\
Grain size & Amorphous pyroxene \\
Composition &
\end{tabular}

values of the two pole directions agree within $1 \sigma$, but the Dec values are currently not compatible even at the $3 \sigma$ level. Because the HST solution (shape model + pole orientation) provides a better fit to the MIPS thermal light curve and to the HST visible light curve (Lamy et al. 2008a), it would be interesting to reanalyze the radar observations of Harmon et al. (2010) with this solution. A shape model and a pole solution that would be consistent with the radar, HST, and SST observations together may then be found.

The radius of the nucleus is more than 2.5 times smaller than expected before the perihelion passage $(7.3 \mathrm{~km}$, Sect. 1), which is quite surprising. We note that a $7.3 \mathrm{~km}$ radius is ruled out by our IRS and MIPS observations in any case because the infrared flux of such a large body would exceed the observed flux, even assuming no coma. The most likely explanation is that although noted as inactive at the time of observation with a stellar profile, the contributions of the coma and of the dust tail to the central pixel were not negligible during earlier ground-based observations.

Finally, our observations are consistent with the bilobate shape of the nucleus of comet 8P/Tuttle. As noted in Sect. 1, this shape is likely common among comets because it was found for four out of the six comets for which we have spatially resolved images. This is also the case of the trans-Neptunian object 2014 $\mathrm{MU}_{69}$ (Ultima Thule) observed by the New Horizon spacecraft (Stern et al. 2019). This binary configuration has some implications for the formation and evolution of 8P/Tuttle. A contact binary could result from (i) the accretion at low velocity of two primordial objects (Massironi et al. 2015; Davidsson et al. 2016), (ii) the disruption of a monolithic object due to excessive spin-up resulting from non-gravitational forces or YORP ${ }^{5}$ effect followed by a reaccretion (Boehnhardt 2004; Ćuk 2007; Hirabayashi et al. 2016), or (iii) the catastrophic disruption of a monolithic object by a collision followed by a re-accretion (Jutzi \& Benz 2017; Schwartz et al. 2018). On the one hand, with a low thermal inertia compared with NEAs, the YORP effect is low for comets, in particular for NIC, which have an elongated orbit and spend most of their time far from the Sun, and it may not be sufficient

5 Yarkovsky-O'Keefe-Radzievskii-Paddack. to increase the spin rate of the nucleus to the point where centrifugal exceed gravitational forces. On the other hand, comet $8 \mathrm{P} /$ Tuttle has been on a very stable orbit for centuries, and it is likely an evolved comet, as suggested by its low activity, so that it could have been much more active in the past. For cometary nuclei, the primary cause for spin-up is torques caused by outgassing, therefore it is possible that $8 \mathrm{P} /$ Tuttle formed as a monolithic body and became a contact binary after its injection into the inner Solar System as a result of excessive spin-up resulting from non-gravitational forces. This scenario has been proposed for comet 67P/Churyumov-Gerasimenko by Hirabayashi et al. (2016). Alternatively, if the binary nature of comet 8P/Tuttle is the result of a primordial accretion or a catastrophic collision in the early Solar Sytem, it could have persisted until now. Similar examples are offered by some binary asteroids that can be stable over the age of the Solar System (Chauvineau et al. 1991), or as proposed by Davidsson et al. (2016) for comet 67P/ChuryumovGerasimenko. For comet 8P/Tuttle, it is however not possible to distinguish the solution of a binary nucleus that formed in the first billion years of our Solar System (e.g., Matonti et al. 2019) from a more recent origin following its injection into the inner Solar System (e.g., Hirabayashi et al. 2016).

Acknowledgements. This work is based on observations made with the Spitzer Space Telescope, which is operated by the Jet Propulsion Laboratory, California Institute of Technology under a contract with NASA. We thank the SST ground system personnel for their prompt and efficient scheduling of the observations. We are grateful to G. Faury (IRAP, Toulouse, France) for his early contribution to the rotational analysis. I.T. was supported by a grant from CNES (Centre National d'Etudes Spatiales) for his work at Laboratoire d'Astrophysique de Marseille. H.W. gratefully acknowledges support provided by NASA through grant number GO-11226 from the Space Telescope Science Institute, which is operated by the Association of Universities for Research in Astronomy under NASA contract NAS5-26555. I.T. acknowledges the support from project GINOP-2.3.2-15-2016-00003 "Cosmic effects and hazards". This research made use of Tiny Tim/Spitzer, developed by John Krist for the Spitzer Science Center. The Center is managed by the California Institute of Technology under a contract with NASA. Finally, we thank D. Wooden for reviewing this paper and for her constructive report.

\section{References}

A'Hearn, M. F., Schleicher, D. G., Millis, R. L., Feldman, P. D., \& Thompson, D. T. 1984 , AJ, 89, 579

A’Hearn, M. F., Millis, R. C., Schleicher, D. O., Osip, D. J., \& Birch, P. V. 1995, Icarus, 118, 223

A'Hearn, M. F., Belton, M. J. S., Delamere, W. A., et al. 2011, Science, 332, 1396

A'Hearn, M. F., Feaga, L. M., Keller, H. U., et al. 2012, ApJ, 758, 29

Barber, R. J., Miller, S., Dello Russo, N., et al. 2009, MNRAS, 398, 1593

Biver, N., Lis, D. C., Fray, N., et al. 2008, LPI Contrib., 1405, 8151

Bockelée-Morvan, D., Crovisier, J., Mumma, M. J., \& Weaver, H. A. 2004, Comets II, The Composition of Cometary Volatiles, eds. M. C. Festou, H. U. Keller, \& H. A. Weaver (Tucson: University of Arizona Press), 391

Bockelée-Morvan, D., Dello Russo, N., Jehin, E., et al. 2008, LPI Contrib., 1405, 8190

Bockelée-Morvan, D., Woodward, C. E., Kelley, M. S., \& Wooden, D. H. 2009, ApJ, 696, 1075

Boehnhardt, H. 2004, Split Comets, eds. M. C. Festou, H. U. Keller, \& H. A. Weaver (Tucson, AZ: University of Arizona), 301

Böhnhardt, H., Mumma, M. J., Villanueva, G. L., et al. 2008, ApJ, 683, L71

Boissier, J., Groussin, O., Jorda, L., et al. 2011, A\&A, 528, A54

Bonev, B. P., Mumma, M. J., Radeva, Y. L., et al. 2008, ApJ, 680, L61

Buratti, B. J., Hicks, M. D., Soderblom, L. A., et al. 2004, Icarus, 167, 16

Chauvineau, B., Mignard, F., \& Farinella, P. 1991, Icarus, 94, 299

Crovisier, J., Leech, K., Bockelee-Morvan, D., et al. 1997, Science, 275, 1904

Ćuk, M. 2007, ApJ, 659, L57

Davidsson, B. J. R., Gutiérrez, P. J., Groussin, O., et al. 2013, Icarus, 224, 154

Davidsson, B. J. R., Sierks, H., Güttler, C., et al. 2016, A\&A, 592, A63

Fernández, Y. R., Kelley, M. S., Lamy, P. L., et al. 2013, Icarus, 226, 1138

Gicquel, A., Bockelée-Morvan, D., Zakharov, V. V., et al. 2012, A\&A, 542, A119 
Groussin, O., Lamy, P., \& Jorda, L. 2004, A\&A, 413, 1163

Groussin, O., A'Hearn, M. F., Li, J.-Y., et al. 2007, Icarus, 187, 16

Groussin, O., Sunshine, J. M., Feaga, L. M., et al. 2013, Icarus, 222, 580

Groussin, O., Attree, N., Brouet, Y., et al. 2019, Space Sci. Rev., 215, 29

Harmon, J. K., Nolan, M. C., Giorgini, J. D., \& Howell, E. S. 2010, Icarus, 207, 499

Harris, A. W. 1998, Icarus, 131, 291

Hirabayashi, M., Scheeres, D. J., Chesley, S. R., et al. 2016, Nature, 534, 352

Houck, J. R., Roellig, T. L., van Cleve, J., et al. 2004, ApJS, 154, 18

Hui, M.-T., \& Li, J.-Y. 2018, PASP, 130, 104501

Jehin, E., Bockelée-Morvan, D., Dello Russo, N., et al. 2009, Earth Moon Planets, 105, 343

Jutzi, M., \& Benz, W. 2017, A\&A, 597, A62

Keller, H. U., Delamere, W. A., Reitsema, H. J., Huebner, W. F., \& Schmidt H. U. 1987, A\&A, 187, 807

Kelley, M. S., \& Wooden, D. H. 2009, Planet. Space Sci., 57, 1133

Kelley, M. S., Fernández, Y. R., Licandro, J., et al. 2013, Icarus, 225, 475

Kobayashi, H., Bockelée-Morvan, D., Kawakita, H., et al. 2010, A\&A, 509, A80

Lagerros, J. S. V. 1998, A\&A, 332, 1123

Lamy, P. L., Toth, I., Fernandez, Y. R., \& Weaver, H. A. 2004, Comets II, eds M. C. Festou, H. U. Keller, \& H. A. Weaver (Tucson: University of Arizona Press), 223

Lamy, P. L., Toth, I., Jorda, L., et al. 2008a, BAAS, 40, 393

Lamy, P. L., Toth, I., Groussin, O., et al. 2008b, A\&A, 489, 777

Lamy, P. L., Jorda, L., Fornasier, S., et al. 2008c, A\&A, 487, 1187

Lebofsky, L. A., \& Spencer, J. R. 1989, Asteroids II, eds. R. P. Binzel, T. Gehrels, \& M. S. Matthews (Tuson: University of Arizona Press), 128

Lebofsky, L. A., Sykes, M. V., Tedesco, E. F., et al. 1986, Icarus, 68, 239
Levison, H. F. 1991, AJ, 102, 787

Levison, H. F., \& Duncan, M. J. 1994, Icarus, 108, 18

Levison, H. F., \& Duncan, M. J. 1997, Icarus, 127, 13

Levison, H. F., Dones, L., \& Duncan, M. J. 2001, AJ, 121, 2253

Licandro, J., Tancredi, G., Lindgren, M., Rickman, H., \& Hutton, R. G. 2000 , Icarus, 147,161

Lippi, M., Mumma, M. J., Villanueva, G. L., et al. 2008, LPI Contrib., 1405, 8197

Lisse, C. M., A'Hearn, M. F., Groussin, O., et al. 2005, ApJ, 625, L139

Lisse, C. M., VanCleve, J., Adams, A. C., et al. 2006, Science, 313, 635

Lovell, A. J., \& Howell, E. S. 2008, LPI Contrib., 1405, 8379

Marshall, D., Groussin, O., Vincent, J.-B., et al. 2018, A\&A, 616, A122

Massironi, M., Simioni, E., Marzari, F., et al. 2015, Nature, 526, 402

Matonti, C., Attree, N., Groussin, O., et al. 2019, Nature Geosci., 12, 157

Reach, W. T., Vaubaillon, J., Lisse, C. M., Holloway, M., \& Rho, J. 2010, Icarus, 208, 276

Rieke, G. H., Young, E. T., Engelbracht, C. W., et al. 2004, ApJS, 154, 25 Schleicher, D. 2007, IAU Circ., 8903

Schloerb, F. P., Keihm, S., von Allmen, P., et al. 2015, A\&A, 583, A29

Schwartz, S. R., Michel, P., Jutzi, M., et al. 2018, Nat. Astron., 2, 379

Sierks, H., Barbieri, C., Lamy, P. L., et al. 2015, Science, 347, 1044

Soderblom, L. A., Becker, T. L., Bennett, G., et al. 2002, Science, 296, 1087

Stern, S. A., Weaver, H. A., Spencer, J. R., et al. 2019, Science, 364, 9771

Weissman, P. R., Choi, Y. J., \& Lowry, S. C. 2008, BAAS, 40, 387

Werner, M. W., Roellig, T. L., Low, F. J., et al. 2004, ApJS, 154, 1

Wooden, D. H., Harker, D. E., \& Brearley, A. J. 2005, ASP Conf. Ser., 341, 774

Wooden, D. H., Ishii, H. A., \& Zolensky, M. E. 2017, Phil. Trans. R. Soc. London, Ser. A, 375, 20160260 